\title{
A Two-Agent Single-Machine Scheduling Problem with Learning and Deteriorating Considerations
}

\author{
Wen-Hsiang Wu \\ Department of Healthcare Management, Yuanpei University, No. 306 Yuanpei St., Hsin Chu 30015, Taiwan \\ Correspondence should be addressed to Wen-Hsiang Wu; wenhsiang_wu@yahoo.com.tw
}

Received 26 May 2013; Accepted 1 July 2013

Academic Editor: Chin-Chia Wu

Copyright (c) 2013 Wen-Hsiang Wu. This is an open access article distributed under the Creative Commons Attribution License, which permits unrestricted use, distribution, and reproduction in any medium, provided the original work is properly cited.

\begin{abstract}
Recently, interest in scheduling with deteriorating jobs and learning effects has kept growing. However, research in this area has seldom considered the multiagent setting. Motivated by these observations, we consider two-agent scheduling on a single machine involving the learning effects and deteriorating jobs simultaneously. In the proposed model, we assume that the actual processing time of a job of the first (second) agent is a decreasing (increasing) function of the total processing time of the jobs already processed in a schedule. The objective is to minimize the total weighted completion time of the jobs of the first agent with the restriction that no tardy job is allowed for the second agent. We develop a branch-and-bound and a simulated annealing algorithms for the problem. We perform extensive computational experiments to test the performance of the algorithms.
\end{abstract}

\section{Introduction}

In classical scheduling, researchers routinely assume that the job processing time is known and fixed from the processing of the first job to the completion of the last job. This assumption is invalid in situations where the job processing time may be prolonged due to deterioration or shortened due to learning over time. For example, Browne and Yechiali [1] observed that the time and effort required to put out a fire increase if there is a delay in the starting of the firefighting effort. In such environments, a job that is processed later consumes more time than the same job when processed earlier. Scheduling in this setting is known as "scheduling deteriorating jobs." Meanwhile, Biskup [2] points out that the repeated processing of similar tasks improves the workers' skills; for example, workers are able to perform setups, deal with machine operations or software, or handle raw materials and components at a faster pace. This phenomenon is known as the "learning effect" in the literature.

The deteriorating job scheduling problem was first introduced by J. N. D. Gupta and S. K. Gupta [3] and Browne and Yechiali [1], independently. J. N. D. Gupta and S. K. Gupta [3] considered the problem using the polynomial processing time functions to minimize the makespan and propose branch-and-bound and heuristic algorithms to search for the optimal and near-optimal solutions. Browne and Yechiali [1] studied the problem using the exponential job processing times to minimize the makespan and provide insights into problem solutions. Since then, an abundance of studies of the subject have emerged. For different models of the problem dealing with different criteria, we refer readers to Alidaee and Womer [4] and Cheng et al. [5].

On the other hand, Biskup [2] and Cheng and Wang [6], independently, incorporate the concept of learning into scheduling. Many researchers have since devoted large amounts of effort to this relatively young but booming area of scheduling research. For detailed reviews of motivations, results, and applications of scheduling with learning effects, we refer the reader to a comprehensive review of scheduling research with learning considerations by Biskup [7].

Recently, there is a growing interest in scheduling research that considers deteriorating jobs and learning effects simultaneously. Wang [8] assumes that the job processing times has the following form: $p_{j[r]}=\left(\alpha_{j}+\beta t\right) r^{a}$, where $p_{j[r]}$ is the actual processing time of a job $J_{j}$ scheduled in the $r$ th position of a sequence, $\alpha_{j}$ is the basic processing time, and $\beta$ 
is the common deteriorating rate. Wang [9] studies a model in which the job processing time has the following form: $p_{j[r]}=p_{j}\left(\alpha(t)+\beta r^{a}\right)$, where $p_{j}$ is the basic processing time and $\alpha(t)$ is an increasing deterioration function with $\alpha(0) \geq 0$. Wang and Cheng [10] consider a model in which the actual processing time is $\left(p_{0}+\alpha_{j} t\right) r^{a}$, where $p_{0}$ is the common basic processing time, $\alpha_{j}$ is the growth rate, and $a$ is the learning index. Cheng et al. [11, 12] study a new scheduling model with deteriorating jobs and learning effects in which the actual processing time of a job $J_{j}$ scheduled in the $r$ th position of a sequence is modeled as $p_{j[r]}=p_{j}\left(\left(p_{0}+\sum_{l=1}^{r-1} p_{[l]}\right) /\left(p_{0}+\sum_{l=1}^{n} p_{l}\right)\right)^{a_{1}} r^{a_{2}}$, where $p_{[l]}$ denotes the normal processing time of the job scheduled in thelth position of the sequence, $p_{0}>0$ is a given parameter, and $a_{1}$ and $a_{2}$ denote the deteriorating and learning indices with $a_{1}<0$ and $a_{2}<0$. Toksari et al. [13] consider several scheduling problems under the assumption of the nonlinear effects of learning and deterioration, where they assume that $p_{j[r]}=\left(p_{[r]}+\alpha t_{[r]}^{b}\right)\left(1+\sum_{k=1}^{r-1} p_{[k]}\right)^{a}, a<0, \alpha>0, b>0$, where $t_{[r]}>0$ is the starting time of the job scheduled in position $r$. Huang et al. [14] consider the single-machine scheduling problem with time-dependent deterioration and an exponential learning effect, that is, the actual processing time of a job depends not only on the processing times of the jobs already processed but also on its scheduled position. Cheng [15] modeled that the actual processing time of $J_{j}$ is defined as $p_{j} \max \left\{\left(1-\left(\left(\sum_{l=1}^{r-1} p_{[l]}\right) /\left(\sum_{l=1}^{n} p_{l}\right)\right)\right)^{a}, \beta\right\}$ if it is scheduled in the $r$ th position in a schedule, where $a \geq 1$ and $0<\beta<1$. Li and Hsu [16] modeled that the real processing time $p_{i r}^{X}$ of job $J_{i}^{X}$ varies with position $r$ based on the learning effect, that is $p_{i r}^{X}=p_{i}^{X} r^{a}$, where $a$ is the learning ratio with $a<0$ and $r=1,2, \ldots, n$.

In classical scheduling, it is assumed that there is a single customer (i.e., agent) who seeks to minimize a scheduling criterion that is function of the order in which the customer's orders (i.e., jobs) are processed by the available processing resources (i.e., machines). In many management situations, however, multiple agents compete on the usage of common processing resources. For instance, Agnetis et al. [17] observe that multiple agents compete on the usage of a common processing resource in different application environments and different methodological fields, such as artificial intelligence, decision theory, and operations research. One major stream of research in this context is related to multiagent scheduling, in which different agents interact to perform their respective tasks, negotiating among one another for the usage of the common resources over time. For research on multiagent scheduling without learning or deteriorating jobs or both, the reader may refer to Baker and Smith [18], Agnetis et al. [19], Yuan et al. [20], Cheng et al. [21], Ng et al. [22], Agnetis et al. [17], Cheng et al. [11, 12], Yin et al. [23], Cheng et al. [24], and so forth. Scheduling in the multiagent setting provides the first motivation for this paper.

Another motivation is that research on multiagent scheduling with deteriorating jobs or learning effects is relatively limited. Liu and Tang's study [25] is probably the only scheduling study that considers deteriorating jobs and multiple agents. They assume that the actual processing time of $J_{j}$ is $\delta_{j} t$, where $\delta_{j}>0$ denotes the deterioration rate and $t$ is the job's starting time. Under the proposed model, they consider the scheduling objectives of minimizing the makespan, maximum lateness, maximum cost, and total completion time. In this paper, we assume that the actual processing time of a job of the first agent is a decreasing function of the total processing time of the jobs already processed in a schedule, while the actual processing time of a job of the second agent is an increasing function of the total processing time of the jobs already processed in a schedule. The objective is to minimize the total weighted completion time of the jobs of the first agent with the restriction that no tardy job is allowed for the second agent.

The remainder of this paper is organized as follows. In Section 2, we present some dominant properties and develop a lower bound to speed up the search for the optimal solution, followed by discussions of branch-and-bound and simulated annealing (SA) algorithm Section 3. We present the results of extensive computational experiments to assess the performance of all of the proposed algorithms under different experimental conditions in Section 4. We conclude the paper and suggest topics for further research in the Section 5.

\section{Problem Statement}

We formulate the problem under study in the following. There are $n$ jobs ready to be processed on a single machine. Each job belongs to one of the two agents, namely, $A G_{0}$ and $A G_{1}$. Associated with job $J_{j}$, there is a normal processing time $p_{j}$, a weight $w_{j}$, a due date $d_{j}$, and an agent code $I_{j}$, where $I_{j}=0$ if $J_{j} \in A G_{0}$, or $I_{j}=1$ if $J_{j} \in A G_{1}$. We assume that all the jobs of $A G_{0}$ have a learning rate $\alpha$ with $\alpha>1$, while all the jobs of $A G_{1}$ have a deteriorating rate $\beta$ with $\beta<0$. Under the proposed model, the actual processing time of $J_{j}$ is $p_{j}\left(1-\left(\left(\sum_{l=1}^{r-1} p_{[l]}\right) /\left(\sum_{l=1}^{n} p_{l}\right)\right)\right)^{a}$ $\left(p_{j}\left(1-\left(\left(\sum_{l=1}^{r-1} p_{[l]}\right) /\left(\sum_{l=1}^{n} p_{l}\right)\right)\right)^{\beta}\right)$ if it is a job of $A G_{0}\left(A G_{1}\right)$ scheduled in the $r$ th position of a sequence, where the subscript $[l]$ denotes the job in the $l$ th position of a sequence. For a given schedule $S$, let $C_{j}(S)$ be the completion time of $J_{j}$ and let $L_{j}(S)=C_{j}(S)-d_{j}$ be the lateness of $J_{j}$. The objective of the scheduling problem is to find an optimal schedule to minimize $\sum_{j=1}^{n} w_{j} C_{j}(S)\left(1-I_{j}\right)$ subject to $\left.\max _{1 \leq j \leq n}\left\{L_{j}(S) I_{j}\right)\right\} \leq 0$. Since the objective function and constraint involve regular scheduling criteria, we use the terms schedule and sequence interchangeably.

\section{Branch-and-Bound and Simulated Annealing Algorithms}

$\mathrm{Ng}$ et al. [22] show that our problem without learning or deteriorating consideration is strongly NP-hard. So, we apply branch-and-bound and SA algorithms to search for the optimal and near-optimal solutions, respectively, in this paper. In order to speed up the searching process, we first develop three adjacent pairwise interchange properties, followed by two dominant rules. We then present the procedures of the branch-and-bound and SA algorithms. 
3.1. Dominant Properties. Assume that the schedule (sequence) $S_{1}$ has two adjacent jobs $J_{i}$ and $J_{j}$ with $J_{i}$ immediately preceding $J_{j}$ and that $J_{i}$ and $J_{j}$ are in the $r$ th and the $(r+1)$ th positions of $S_{1}$, respectively. Perform a pairwise interchange of $J_{i}$ and $J_{j}$ to derive a new sequence $S_{2}$.

Proposition 1. For any two jobs $J_{i}$ and $J_{j} \in A G_{0}$ to be scheduled consecutively, if $p_{j} / p_{i} \geq w_{j} / w_{i}>1$, then $S_{1}$ dominates $S_{2}$.

Proof. Assume that $S_{1}=\left(\pi, J_{i}, J_{j}, \pi^{\prime}\right)$ and $S_{2}=\left(\pi, J_{j}, J_{i}, \pi^{\prime}\right)$ denote two sequences in which $\pi$ and $\pi^{\prime}$ denote partial sequences. To show that $S_{1}$ dominates $S_{2}$, it suffices to show that $\left[w_{i} C_{i}\left(S_{1}\right)+w_{j} C_{j}\left(S_{1}\right)\right] \leq\left[w_{j} C_{j}\left(S_{2}\right)+w_{i} C_{i}\left(S_{2}\right)\right]$ and $C_{j}\left(S_{1}\right)<C_{i}\left(S_{2}\right)$. In addition, let $A$ be the completion time of the last job in the subsequence $\pi$ with $(r-1)$ jobs. Since $J_{i}$ and $J_{j} \in A G_{0}$, the completion time of the jobs $J_{i}$ and $J_{j}$ in sequences $S_{1}$ and $S_{2}$ is given by the following:

$$
\begin{aligned}
C_{i}\left(S_{1}\right)= & A+p_{i}\left(1-\frac{\sum_{l=1}^{r-1} p_{[l]}}{\sum p_{i}}\right)^{a}, \\
C_{j}\left(S_{1}\right)= & A+p_{i}\left(1-\frac{\sum_{l=1}^{r-1} p_{[l]}}{\sum p_{i}}\right)^{a} \\
& +p_{j}\left(1-\frac{\sum_{l=1}^{r-1} p_{[l]}+p_{i}}{\sum p_{i}}\right)^{a}, \\
C_{j}\left(S_{2}\right)= & A+p_{j}\left(1-\frac{\sum_{l=1}^{r-1} p_{[l]}}{\sum p_{i}}\right)^{a} .
\end{aligned}
$$

One also has

$$
\begin{aligned}
C_{i}\left(S_{2}\right)= & A+p_{j}\left(1-\frac{\sum_{l=1}^{r-1} p_{[l]}}{\sum p_{i}}\right)^{a} \\
& +p_{i}\left(1-\frac{\sum_{l=1}^{r-1} p_{[l]}+p_{j}}{\sum p_{i}}\right)^{a} .
\end{aligned}
$$

Taking the difference between $S_{1}$ and $S_{2}$, we obtain the following:

$$
\begin{aligned}
& {\left[w_{j} C_{j}\left(S_{2}\right)+w_{i} C_{i}\left(S_{2}\right)\right]-\left[w_{i} C_{i}\left(S_{1}\right)+w_{j} C_{j}\left(S_{1}\right)\right]} \\
& =\left(w_{i} p_{j}-w_{j} p_{i}\right)\left(1-\frac{\sum_{l=1}^{r-1} p_{[l]}}{\sum p_{i}}\right)^{a} \\
& +w_{j} p_{j}\left[\left(1-\frac{\sum_{l=1}^{r-1} p_{[l]}}{\sum p_{i}}\right)^{a}-\left(1-\frac{\sum_{l=1}^{r-1} p_{[l]}+p_{i}}{\sum p_{i}}\right)^{a}\right] \\
& -w_{i} p_{i}\left[\left(1-\frac{\sum_{l=1}^{r-1} p_{[l]}}{\sum p_{i}}\right)^{a}-\left(1-\frac{\sum_{l=1}^{r-1} p_{[l]}+p_{j}}{\sum p_{i}}\right)^{a}\right] .
\end{aligned}
$$

By substituting $\theta=\left(p_{j} / w_{j}\right) /\left(p_{i} / w_{i}\right), c=w_{j} / w_{i}$, and $x=$ $p_{i} /\left(\sum p_{i}-\sum_{l=1}^{r-1} p_{[l]}\right)$, we simplify (3) as follows:

$$
\begin{aligned}
{\left[w_{j} C_{j}\right.} & \left.\left(S_{2}\right)+w_{i} C_{i}\left(S_{2}\right)\right]-\left[w_{i} C_{i}\left(S_{1}\right)+w_{j} C_{j}\left(S_{1}\right)\right] \\
= & w_{j} p_{i}\left(1-\frac{\sum_{l=1}^{r-1} p_{[l]}}{\sum p_{i}}\right)^{a} \\
& \times\left\{(\theta-1)+c \theta\left[1-(1-x)^{a}\right]-\frac{1}{c}\left[1-(1-c \theta x)^{a}\right]\right\},
\end{aligned}
$$

where $c \geq 1, \theta \geq 1, a>1$, and $0<x<1$. Taking the first and second derivatives of (4), we can see that (4) is nonnegative. Hence, we have

$$
\left[w_{j} C_{j}\left(S_{2}\right)+w_{i} C_{i}\left(S_{2}\right)\right] \geq\left[w_{i} C_{i}\left(S_{1}\right)+w_{j} C_{j}\left(S_{1}\right)\right] .
$$

On the other hand, taking the difference between (1) and (2), we have

$$
\begin{aligned}
C_{i}\left(S_{2}\right)-C_{j}\left(S_{1}\right)= & \left(p_{j}-p_{i}\right)\left(1-\frac{\sum_{l=1}^{r-1} p_{[l]}}{\sum_{l=1}^{n} p_{l}}\right)^{a} \\
& +p_{i}\left(1-\frac{\sum_{l=1}^{r-1} p_{[l]}+p_{j}}{\sum_{l=1}^{n} p_{l}}\right)^{a} \\
& -p_{j}\left(1-\frac{\sum_{l=1}^{r-1} p_{[l]}+p_{i}}{\sum_{l=1}^{n} p_{l}}\right)^{a}
\end{aligned}
$$

By substituting $\theta=p_{j} / p_{i}, u=\left(1-\left(\left(\sum_{l=1}^{r-1} p_{[l]}\right) /\left(\sum_{l=1}^{n} p_{l}\right)\right)\right)$, and $x=\left(p_{i} / \sum_{l=1}^{n} p_{l}\right)$ into $(6)$, we have

$$
C_{i}\left(S_{2}\right)-C_{j}\left(S_{1}\right)=p_{i} u^{a}\left[(\theta-1)+(1-\theta x)^{a}-\theta(1-x)^{a}\right] .
$$

Taking the first and second derivatives of (7), and noting that $\theta>1, a>1$, and $0<x<1$, we have $C_{i}\left(S_{2}\right)-C_{j}\left(S_{1}\right)>0$. Therefore, $S_{1}$ dominates $S_{2}$.

Proposition 2. For any two jobs $J_{i}$ and $J_{j} \in A G_{1}$ to be scheduled consecutively, if $p_{i}<p_{j}, A+p_{i}\left(1-\left(\left(\sum_{l=1}^{r-1} p_{[l]}\right) /\left(\sum_{l=1}^{n} p_{l}\right)\right)\right)^{\beta}$ $+p_{j}\left(1-\left(\left(\sum_{l=1}^{r-1} p_{[l]}+p_{i}\right) /\left(\sum_{l=1}^{n} p_{l}\right)\right)\right)^{\beta}<d_{j}$, and $A+p_{i}$ $\left(1-\left(\left(\sum_{l=1}^{r-1} p_{[l]}\right) /\left(\sum_{l=1}^{n} p_{l}\right)\right)\right)^{\beta}<d_{i}$, then $S_{1}$ dominates $S_{2}$.

Proposition 3. For job $J_{i} \in A G_{1}$ and job $J_{j} \in A G_{0}$ to be scheduled consecutively, if $A+p_{i}\left(1-\left(\left(\sum_{l=1}^{r-1} p_{[l]}\right) /\left(\sum_{l=1}^{n} p_{l}\right)\right)\right)^{\beta}<d_{i}$ and $p_{i}<p_{j}\left[\left(1-\left(\left(\sum_{l=1}^{r-1} p_{[l]}\right) /\left(\sum_{l=1}^{n} p_{l}\right)\right)\right)^{a}-\left(1-\left(\left(\sum_{l=1}^{r-1} p_{[l]}\right.\right.\right.\right.$ $\left.\left.\left.\left.+p_{i}\right) /\left(\sum_{l=1}^{n} p_{l}\right)\right)\right)^{a}\right] /\left(1-\left(\left(\sum_{l=1}^{r-1} p_{[l]}\right) /\left(\sum_{l=1}^{n} p_{l}\right)\right)\right)^{\beta}$, then $S_{1}$ dominates $S_{2}$.

We omit the proofs of Propositions 2 and 3 because they are similar to that of Proposition 1.

We next present two propositions to determine the feasibility of a partial sequence. Let $\left(\pi, \pi^{c}\right)$ be a sequence of 
jobs, where $\pi$ is the scheduled part with $k$ jobs and $\pi^{c}$ is the unscheduled part. Moreover, let $C_{[k]}$ be the completion time of the last job in $\pi$.

Proposition 4. If there is a job $J_{j} \in A G_{1} \cap \pi^{c}$ such that $C_{[k]}>$ $d_{j}$, then sequence $\left(\pi, \pi^{c}\right)$ is not a feasible solution.

Proof. If there is a job $J_{j} \in A G_{1} \cap \pi^{c}$ such that $C_{[k]}>d_{j}$, this violates the constraint that no tardy job is allowed for the second agent.

Proposition 5. If there is a job $J_{j} \in A G_{1} \cap \pi^{c}$ such that $C_{[k]}+$ $p_{j}\left(1-\left(\left(\sum_{l=1}^{k} p_{[l]}\right) /\left(\sum_{l=1}^{n} p_{l}\right)\right)\right)^{\beta}>d_{j}$, then sequence $\left(\pi, \pi^{c}\right)$ is not a feasible solution.

Proof. Similar to Proposition 4.

3.2. Lower Bound. In this subsection, we develop a lower bound for the proposed branch-and-bound algorithm. Suppose that $P S$ is a partial schedule in which the order of the first $k$ jobs is determined, and, US be the unscheduled part with $(n-k)$ jobs, where there are $n_{0}$ jobs of $A G_{0}$ and $n_{1}$ jobs of $A G_{1}$ with $n_{0}+n_{1}=n-k$. Moreover, let $p_{(k+1)} \leq p_{(k+2)} \leq$ $\cdots \leq p_{(n)}$ denote the normal processing time of the $(n-k)$ unscheduled jobs when arranged in nondecreasing order, and let $C_{[k]}$ be the completion time of the last job in PS. Given that the learning effect can shorten the processing time and that the deteriorating effect can lengthen the processing time, we assign the jobs with the learning effect to the first $n_{0}$ positions and the jobs with the deteriorating effect to the following $n_{1}$ positions of the remaining $(n-k)$ unscheduled positions after the $k$ th position which contributes in the reduction the total weighted completion time of the jobs of the first agent. The completion time of the $(k+1)$ th job is

$$
\begin{aligned}
& C_{[k+1]} \\
& =C_{[k]}+p_{[k+1]}\left[\left(1-\frac{\sum_{l=1}^{k} p_{[l]}}{\sum_{l=1}^{n} p_{l}}\right)^{a} I_{\{[k+1]\}}\right. \\
& \left.+\left(1-\frac{\sum_{l=1}^{k} p_{[l]}}{\sum_{l=1}^{n} p_{l}}\right)^{\beta}\left(1-I_{\{[k+1]\}}\right)\right] \\
& \geq C_{[k]}+p_{(k+1)}\left(1-\frac{\sum_{l=1}^{k} p_{[l]}}{\sum_{l=1}^{n} p_{l}}\right)^{a} .
\end{aligned}
$$

Similarly, the completion time of the $(k+j)$ th job is

$$
\begin{aligned}
& C_{[k+j]} \geq C_{[k]}+p_{(k+1)}\left(1-\frac{\sum_{l=1}^{k} p_{[l]}}{\sum_{l=1}^{n} p_{l}}\right)^{a} \\
&+\sum_{i=2}^{j} p_{(k+i)}\left(1-\frac{\sum_{l=1}^{k} p_{[l]}+\sum_{l=2}^{j} p_{(n-l+2)}}{\sum_{l=1}^{n} p_{l}}\right)^{a}, \\
& 2 \leq j \leq n_{0} .
\end{aligned}
$$

Next, we deriequence by arranging the jobs ofe the completion time of the jobs with the deteriorating effect assigned to the remaining $n_{1}$ positions. The completion time of the $\left(k+n_{0}+j\right)$ th job is

$$
\begin{aligned}
& C_{\left[k+n_{0}+j\right]} \geq C_{\left[k+n_{0}\right]} \\
&+ \sum_{i=1}^{j} p_{(k+1)}\left(1-\frac{\sum_{l=1}^{k} p_{[l]}+\sum_{l=1}^{j-1} p_{(k+l)}}{\sum_{l=1}^{n} p_{l}}\right)^{\beta}, \\
& \text { for } 1 \leq j \leq n_{1} .
\end{aligned}
$$

Note that $\sum_{l=1}^{0} p_{(k+l)}=0$ and

$$
\begin{aligned}
C_{\left[k+n_{0}\right]} \geq & C_{[k]}+p_{(k+1)}\left(1-\frac{\sum_{l=1}^{k} p_{[l]}}{\sum_{l=1}^{n} p_{l}}\right)^{a} \\
& +\sum_{i=2}^{n_{0}} p_{(k+i)}\left(1-\frac{\sum_{l=1}^{k} p_{[l]}+\sum_{l=2}^{j} p_{(n-l+2)}}{\sum_{l=1}^{n} p_{l}}\right)^{a} .
\end{aligned}
$$

Following the same idea of Cheng et al. [26] and Wu et al. [27], we want to assign the job completion time to the jobs of agents $A G_{0}$ and $A G_{1}$. Given the constraint that the jobs of agent $A G_{1}$ cannot be tardy, we assign the completion time to the jobs of agent $A G_{1}$ as late as possible. The procedure is as follows.

\subsubsection{Algorithm}

Step 1. Set

$$
\begin{aligned}
& \widehat{C}_{[k+1]}= C_{[k]}+p_{(k+1)}\left(1-\frac{\sum_{l=1}^{k} p_{[l]}}{\sum_{l=1}^{n} p_{l}}\right)^{a}, \quad \text { for } j=1, \\
& \widehat{C}_{[k+j]}= C_{[k]}+p_{(k+1)}\left(1-\frac{\sum_{l=1}^{k} p_{[l]}}{\sum_{l=1}^{n} p_{l}}\right)^{a} \\
&+\sum_{i=2}^{j} p_{(k+i)}\left(1-\frac{\sum_{l=1}^{k} p_{[l]}+\sum_{l=2}^{j} p_{(n-l+2)}}{\sum_{l=1}^{n} p_{l}}\right)^{a}, \\
& \widehat{C}_{\left[k+n_{0}+j\right]}= \widehat{C}_{\left[k+n_{0}\right]} \text { for } 2 \leq j \leq n_{0}, \\
&+\sum_{i=1}^{j} p_{(k+1)}\left(1-\frac{\sum_{l=1}^{k} p_{[l]}+\sum_{l=1}^{j-1} p_{(k+l)}}{\sum_{l=1}^{n} p_{l}}\right)^{\beta}, \\
& \text { for } 1 \leq j \leq n_{1} .
\end{aligned}
$$

Step 2. Sort the jobs of agent $A G_{0}$ in a nondecreasing order of their weights and the jobs of agent $A G_{1}$ in a nondecreasing order of their due dates, that is,

$$
w_{(1)}^{0} \leq w_{(2)}^{0} \leq \cdots \leq w_{\left(n_{0}\right)}^{0}, \quad d_{(1)}^{1} \leq d_{(2)}^{1} \leq \cdots \leq d_{\left(n_{1}\right)}^{1} .
$$


TABLE 1: Performance of the branch-and-bound and SA algorithms $(n=10$, pro $=0.4)$.

\begin{tabular}{|c|c|c|c|c|c|c|c|c|c|c|c|c|c|c|}
\hline \multirow{3}{*}{ Pro } & \multirow{3}{*}{$\tau$} & \multirow{3}{*}{$R$} & \multirow{3}{*}{$a$} & \multirow{3}{*}{$\beta$} & \multicolumn{4}{|c|}{ Branch-and-bound algorithm } & \multirow{2}{*}{\multicolumn{2}{|c|}{$\mathrm{SA}_{1}$}} & \multirow{2}{*}{\multicolumn{2}{|c|}{$\begin{array}{c}\mathrm{SA}_{2} \\
\text { Error percentages }\end{array}$}} & \multirow{2}{*}{\multicolumn{2}{|c|}{$\mathrm{SA}_{3}$}} \\
\hline & & & & & \multicolumn{2}{|c|}{ CPU time } & \multicolumn{2}{|c|}{ Number of nodes } & & & & & & \\
\hline & & & & & Mean & Std & Mean & Std & Mean & Std & Mean & Std & Mean & Std \\
\hline \multirow[t]{48}{*}{0.4} & \multirow[t]{27}{*}{0.40} & \multirow[t]{9}{*}{0.40} & \multirow[t]{3}{*}{1.100} & -1.100 & 103.98 & 276.35 & 3921045 & 10555474 & 0.543 & 1.381 & 0.530 & 1.452 & 0.247 & 0.883 \\
\hline & & & & -1.010 & 63.93 & 217.06 & 2380521 & 8139948 & 0.223 & 0.659 & 0.409 & 0.924 & 0.197 & 0.658 \\
\hline & & & & -1.001 & 65.47 & 222.48 & 2437016 & 8344800 & 0.216 & 0.507 & 0.165 & 0.483 & 0.170 & 0.381 \\
\hline & & & \multirow[t]{3}{*}{1.010} & -1.100 & 95.82 & 253.71 & 3623888 & 9719538 & 0.478 & 1.500 & 0.397 & 1.219 & 0.298 & 1.126 \\
\hline & & & & -1.010 & 58.55 & 198.37 & 2187372 & 7459183 & 0.272 & 0.725 & 0.239 & 0.630 & 0.182 & 0.586 \\
\hline & & & & -1.001 & 59.55 & 201.41 & 2223379 & 7575433 & 0.271 & 0.671 & 0.322 & 0.897 & 0.145 & 0.416 \\
\hline & & & 1.001 & -1.100 & 95.15 & 251.83 & 3600246 & 9653456 & 0.420 & 1.255 & 0.656 & 1.637 & 0.175 & 0.620 \\
\hline & & & & -1.010 & 58.05 & 196.57 & 2169483 & 7394568 & 0.174 & 0.466 & 0.416 & 1.046 & 0.168 & 0.525 \\
\hline & & & & -1.001 & 59.06 & 199.77 & 2206236 & 7517115 & 0.200 & 0.625 & 0.208 & 0.618 & 0.153 & 0.497 \\
\hline & & 0.50 & 1.100 & -1.100 & 85.80 & 248.97 & 3205789 & 9430455 & 0.527 & 1.543 & 0.227 & 0.569 & 0.210 & 0.455 \\
\hline & & & & -1.010 & 68.71 & 244.97 & 2549809 & 9236732 & 0.581 & 2.138 & 0.318 & 0.891 & 0.171 & 0.419 \\
\hline & & & & -1.001 & 68.38 & 248.11 & 2551177 & 9366715 & 0.692 & 1.981 & 0.161 & 0.386 & 0.347 & 0.856 \\
\hline & & & 1.010 & -1.100 & 79.98 & 232.32 & 2995531 & 8817308 & 0.575 & 1.440 & 0.439 & 1.103 & 0.219 & 0.667 \\
\hline & & & & -1.010 & 63.28 & 226.24 & 2354281 & 8550234 & 0.699 & 1.835 & 0.622 & 1.664 & 0.242 & 0.662 \\
\hline & & & & -1.001 & 62.63 & 227.25 & 2341949 & 8599304 & 0.316 & 0.789 & 0.297 & 0.777 & 0.143 & 0.348 \\
\hline & & & 1.001 & -1.100 & 79.34 & 230.50 & 2972794 & 8751750 & 0.417 & 1.087 & 0.530 & 1.435 & 0.180 & 0.485 \\
\hline & & & & -1.010 & 62.76 & 224.44 & 2335360 & 8482895 & 0.285 & 0.771 & 0.567 & 1.530 & 0.321 & 1.380 \\
\hline & & & & -1.001 & 62.08 & 225.42 & 2321896 & 8531873 & 0.405 & 0.965 & 0.217 & 0.694 & 0.114 & 0.314 \\
\hline & & 0.60 & 1.100 & -1.100 & 75.42 & 256.62 & 2793897 & 9601920 & 0.546 & 1.308 & 0.447 & 0.970 & 0.359 & 1.051 \\
\hline & & & & -1.010 & 64.40 & 245.82 & 2384768 & 9203252 & 0.625 & 1.263 & 0.811 & 2.576 & 0.314 & 0.666 \\
\hline & & & & -1.001 & 65.53 & 248.86 & 2427465 & 9311518 & 0.369 & 0.874 & 0.352 & 0.859 & 0.285 & 0.676 \\
\hline & & & 1.010 & -1.100 & 71.27 & 242.87 & 2648314 & 9106695 & 0.576 & 1.457 & 0.327 & 0.922 & 0.254 & 0.679 \\
\hline & & & & -1.010 & 59.88 & 229.35 & 2225401 & 8623062 & 0.381 & 0.693 & 0.429 & 1.125 & 0.405 & 1.064 \\
\hline & & & & -1.001 & 60.77 & 231.22 & 2258351 & 8680937 & 0.565 & 1.358 & 0.732 & 2.489 & 0.329 & 1.122 \\
\hline & & & 1.001 & -1.100 & 70.71 & 241.02 & 2627453 & 9038121 & 0.360 & 0.943 & 0.357 & 1.033 & 0.135 & 0.320 \\
\hline & & & & -1.010 & 60.15 & 230.09 & 2237063 & 8644312 & 0.809 & 1.726 & 0.531 & 1.307 & 0.378 & 1.006 \\
\hline & & & & -1.001 & 60.20 & 229.00 & 2237648 & 8599211 & 0.567 & 1.440 & 0.462 & 1.334 & 0.266 & 0.614 \\
\hline & 0.50 & 0.40 & 1.100 & -1.100 & 19.94 & 59.21 & 780723 & 2349939 & 0.312 & 1.111 & 0.276 & 0.711 & 0.141 & 0.387 \\
\hline & & & & -1.010 & 30.23 & 77.19 & 1183839 & 3050478 & 0.270 & 0.730 & 0.303 & 1.152 & 0.095 & 0.253 \\
\hline & & & & -1.001 & 30.91 & 78.69 & 1209380 & 3107819 & 1.058 & 6.974 & 0.139 & 0.420 & 0.927 & 6.386 \\
\hline & & & 1.010 & -1.100 & 18.32 & 54.31 & 721468 & 2166990 & 0.249 & 0.619 & 0.201 & 0.522 & 0.080 & 0.245 \\
\hline & & & & -1.010 & 27.57 & 70.55 & 1085563 & 2803070 & 0.202 & 0.511 & 0.355 & 1.077 & 0.480 & 2.424 \\
\hline & & & & -1.001 & 28.17 & 71.84 & 1108207 & 2852184 & 0.850 & 3.491 & 0.273 & 0.751 & 0.187 & 0.844 \\
\hline & & & 1.001 & -1.100 & 18.16 & 53.83 & 715408 & 2149077 & 0.086 & 0.220 & 0.196 & 0.611 & 0.113 & 0.301 \\
\hline & & & & -1.010 & 27.30 & 69.90 & 1075579 & 2778464 & 1.006 & 5.165 & 0.230 & 1.018 & 0.821 & 5.100 \\
\hline & & & & -1.001 & 27.89 & 71.17 & 1097883 & 2827375 & 1.220 & 8.550 & 0.518 & 2.713 & 0.164 & 0.818 \\
\hline & & 0.50 & 1.100 & -1.100 & 27.86 & 73.51 & 1088932 & 2909727 & 0.557 & 2.021 & 0.244 & 0.553 & 0.132 & 0.344 \\
\hline & & & & -1.010 & 27.67 & 74.52 & 1067668 & 2921795 & 0.464 & 1.396 & 0.258 & 0.811 & 0.132 & 0.335 \\
\hline & & & & -1.001 & 27.89 & 76.10 & 1078229 & 2982686 & 0.417 & 1.217 & 0.607 & 1.722 & 0.096 & 0.331 \\
\hline & & & 1.010 & -1.100 & 25.46 & 67.07 & 1000555 & 2665211 & 0.250 & 0.875 & 0.285 & 0.839 & 0.083 & 0.190 \\
\hline & & & & -1.010 & 25.43 & 68.23 & 985378 & 2685319 & 0.512 & 1.747 & 0.380 & 1.148 & 0.158 & 0.697 \\
\hline & & & & -1.001 & 25.70 & 69.91 & 997591 & 2751685 & 0.507 & 1.384 & 0.392 & 1.143 & 0.293 & 1.141 \\
\hline & & & 1.001 & -1.100 & 25.23 & 66.50 & 992131 & 2644048 & 0.681 & 2.156 & 0.217 & 0.807 & 0.216 & 0.815 \\
\hline & & & & -1.010 & 25.20 & 67.61 & 977049 & 2661873 & 0.519 & 1.392 & 0.264 & 0.703 & 0.289 & 0.982 \\
\hline & & & & -1.001 & 25.43 & 69.16 & 987380 & 2722447 & 0.311 & 0.823 & 0.311 & 0.963 & 0.296 & 1.136 \\
\hline & & 0.60 & 1.100 & -1.100 & 32.49 & 83.06 & 1259437 & 3245114 & 0.381 & 1.107 & 0.432 & 1.539 & 0.118 & 0.302 \\
\hline & & & & -1.010 & 22.45 & 74.47 & 847123 & 2812785 & 0.718 & 1.975 & 0.345 & 1.111 & 0.090 & 0.230 \\
\hline & & & & -1.001 & 22.85 & 75.78 & 861566 & 2861344 & 0.295 & 0.705 & 0.598 & 1.635 & 0.151 & 0.324 \\
\hline
\end{tabular}


TABle 1: Continued.

\begin{tabular}{|c|c|c|c|c|c|c|c|c|c|c|c|c|c|c|}
\hline \multirow{3}{*}{ Pro } & \multirow{3}{*}{$\tau$} & \multirow{3}{*}{$R$} & \multirow{3}{*}{$a$} & \multirow{3}{*}{$\beta$} & \multicolumn{4}{|c|}{ Branch-and-bound algorithm } & \multirow{2}{*}{\multicolumn{2}{|c|}{$\mathrm{SA}_{1}$}} & \multirow{2}{*}{\multicolumn{2}{|c|}{$\begin{array}{c}\mathrm{SA}_{2} \\
\text { Error percentages }\end{array}$}} & \multicolumn{2}{|c|}{$\mathrm{SA}_{3}$} \\
\hline & & & & & \multicolumn{2}{|c|}{ CPU time } & \multicolumn{2}{|c|}{ Number of nodes } & & & & & \multirow[b]{2}{*}{ Mean } & \multirow[b]{2}{*}{ Std } \\
\hline & & & & & Mean & Std & Mean & Std & Mean & Std & \multicolumn{2}{|c|}{ Mean Std } & & \\
\hline & \multirow{33}{*}{0.60} & \multirow{15}{*}{0.40} & \multirow[t]{3}{*}{1.010} & -1.100 & 29.96 & 76.43 & 1163916 & 2987954 & 0.535 & 1.425 & 0.378 & 1.250 & 0.098 & 0.269 \\
\hline & & & & -1.010 & 21.02 & 69.88 & 797393 & 2652794 & 0.386 & 1.012 & 0.479 & 1.419 & 0.207 & 0.757 \\
\hline & & & & -1.001 & 21.34 & 70.85 & 808645 & 2687918 & 0.848 & 2.490 & 0.454 & 1.468 & 0.147 & 0.469 \\
\hline & & & \multirow[t]{3}{*}{1.001} & -1.100 & 29.76 & 75.79 & 1156347 & 2963377 & 0.319 & 0.960 & 0.360 & 0.984 & 0.296 & 0.930 \\
\hline & & & & -1.010 & 20.92 & 69.31 & 793687 & 2631768 & 0.455 & 1.079 & 0.642 & 1.865 & 0.182 & 0.696 \\
\hline & & & & -1.001 & 21.16 & 70.27 & 802222 & 2666815 & 0.468 & 1.233 & 0.514 & 1.464 & 0.232 & 0.842 \\
\hline & & & \multirow[t]{3}{*}{1.100} & -1.100 & 1.95 & 5.15 & 79358 & 214347 & 0.311 & 1.439 & 0.105 & 0.295 & 0.124 & 0.376 \\
\hline & & & & -1.010 & 1.73 & 5.65 & 70241 & 235697 & 0.125 & 0.309 & 0.105 & 0.336 & 0.165 & 0.827 \\
\hline & & & & -1.001 & 1.73 & 5.70 & 70552 & 237669 & 0.144 & 0.366 & 0.147 & 0.368 & 0.094 & 0.322 \\
\hline & & & \multirow[t]{3}{*}{1.010} & -1.100 & 1.82 & 4.83 & 74060 & 201604 & 0.330 & 1.453 & 0.262 & 1.441 & 0.125 & 0.389 \\
\hline & & & & -1.010 & 1.57 & 5.10 & 64068 & 213383 & 0.266 & 1.477 & 0.146 & 0.361 & 0.076 & 0.197 \\
\hline & & & & -1.001 & 1.56 & 5.14 & 64003 & 215085 & 0.120 & 0.289 & 0.128 & 0.334 & 0.079 & 0.273 \\
\hline & & & \multirow[t]{3}{*}{1.001} & -1.100 & 1.81 & 4.82 & 73630 & 200978 & 0.150 & 0.360 & 0.144 & 0.324 & 0.102 & 0.292 \\
\hline & & & & -1.010 & 1.55 & 5.05 & 63473 & 211343 & 0.220 & 0.706 & 0.131 & 0.391 & 0.106 & 0.279 \\
\hline & & & & -1.001 & 1.55 & 5.10 & 63463 & 213337 & 0.326 & 1.471 & 0.172 & 0.508 & 0.045 & 0.151 \\
\hline & & 0.50 & 1.100 & -1.100 & 2.32 & 7.36 & 95199 & 309557 & 0.159 & 0.569 & 0.141 & 0.528 & 0.069 & 0.220 \\
\hline & & & & -1.010 & 3.06 & 9.20 & 124875 & 380846 & 0.223 & 0.713 & 0.225 & 0.656 & 0.136 & 0.610 \\
\hline & & & & -1.001 & 3.11 & 9.33 & 127086 & 386185 & 0.363 & 1.193 & 0.334 & 1.068 & 0.089 & 0.289 \\
\hline & & & 1.010 & -1.100 & 2.11 & 6.73 & 86838 & 283413 & 0.270 & 0.875 & 0.132 & 0.481 & 0.179 & 0.805 \\
\hline & & & & -1.010 & 2.78 & 8.44 & 114224 & 351088 & 0.385 & 1.169 & 0.135 & 0.402 & 0.048 & 0.127 \\
\hline & & & & -1.001 & 2.83 & 8.56 & 116276 & 355790 & 0.233 & 0.853 & 0.258 & 0.800 & 0.054 & 0.180 \\
\hline & & & 1.001 & -1.100 & 2.09 & 6.66 & 85990 & 280750 & 0.373 & 1.450 & 0.186 & 0.809 & 0.062 & 0.210 \\
\hline & & & & -1.010 & 2.75 & 8.36 & 113098 & 347937 & 0.112 & 0.354 & 0.229 & 0.793 & 0.046 & 0.125 \\
\hline & & & & -1.001 & 2.80 & 8.48 & 115164 & 352785 & 0.158 & 0.643 & 0.397 & 1.297 & 0.052 & 0.197 \\
\hline & & 0.60 & 1.100 & -1.100 & 3.44 & 10.66 & 139504 & 436210 & 0.145 & 0.546 & 0.293 & 0.936 & 0.059 & 0.206 \\
\hline & & & & -1.010 & 4.91 & 13.22 & 202484 & 547733 & 0.086 & 0.310 & 0.121 & 0.260 & 0.072 & 0.197 \\
\hline & & & & -1.001 & 5.02 & 13.44 & 207022 & 556511 & 0.327 & 1.314 & 0.304 & 1.301 & 0.126 & 0.423 \\
\hline & & & 1.010 & -1.100 & 3.12 & 9.69 & 127253 & 398528 & 0.206 & 0.902 & 0.230 & 0.934 & 0.097 & 0.324 \\
\hline & & & & -1.010 & 4.47 & 12.10 & 185230 & 502913 & 0.303 & 1.447 & 0.079 & 0.212 & 0.047 & 0.187 \\
\hline & & & & -1.001 & 4.57 & 12.29 & 189209 & 510614 & 0.427 & 2.235 & 0.350 & 2.226 & 0.038 & 0.130 \\
\hline & & & 1.001 & -1.100 & 3.09 & 9.61 & 126170 & 395114 & 0.175 & 0.618 & 0.219 & 0.518 & 0.096 & 0.328 \\
\hline & & & & -1.010 & 4.43 & 11.99 & 183664 & 498778 & 0.356 & 1.516 & 0.213 & 0.493 & 0.035 & 0.090 \\
\hline & & & & -1.001 & 4.53 & 12.19 & 187563 & 506370 & 0.306 & 1.461 & 0.428 & 2.235 & 0.087 & 0.286 \\
\hline & Avera & & & & 32.64 & 103.83 & 1234705 & 3960746 & 0.402 & 1.356 & 0.326 & 1.003 & 0.184 & 0.668 \\
\hline
\end{tabular}

Step 3. Set $i c=n, i a=n_{0}$, and $i b=n_{1}$.

Step 4. If $i c \leq n-k$, go to Step 7 .

Step 5. If $\widehat{C}_{[i c]} \leq d_{(i b)}^{1}$, set $C_{(i b)}^{1}=\widehat{C}_{[i c]}$ and $i b=i b-1$. Otherwise, set $C_{(i a)}^{0}=\widehat{C}_{[i c]}$ and $i a=i a-1$.

Step 6. Set $i c=i c-1$, and go to Step 4 .

Step 7. Compute $L B_{U S}=\sum_{j=1}^{n_{0}} w_{\left(n_{0}-j+1\right)}^{0} C_{(j)}^{0}$. Evidently, a lower bound for the partial sequence $P S$ is

$$
L B=\sum_{j \in P S} w_{[j]} C_{[j]}\left(1-I_{j}\right)+L B_{U S} .
$$

3.3. Simulated Annealing Algorithms. Simulated annealing is one of the most popular metaheuristic methods widely applied to solve combinatorial optimization problems [2830]. SA has the advantage of avoiding getting trapped in a local optimum because of its hill climbing moves, which are governed by a control parameter. In the following, we apply SA to derive near-optimal solutions for our problem. We apply an SA algorithm with three different initials as follows.

Step 1. Initial sequence.

We use three initial sequences for the three SA algorithms. In $\mathrm{SA}_{1}$, we use random numbers to generate an initial job sequence; if there is any tardy job in this sequence, we regenerate another job sequence until it is feasible. In $\mathrm{SA}_{2}$, we create an initial job sequence by arranging the jobs of $A G_{1}$ in the earliest due date (EDD) order, followed by arranging the jobs of $A G_{0}$ in the shortest processing time (SPT) order. In $\mathrm{SA}_{3}$, we create an initial job sequence by arranging the jobs of $A G_{1}$ in the EDD order, followed by arranging the jobs of $A G_{0}$ 
TABLE 2: Performance of the branch-and-bound and SA algorithms $(n=10$, pro $=0.6)$.

\begin{tabular}{|c|c|c|c|c|c|c|c|c|c|c|c|c|c|c|}
\hline \multirow{3}{*}{ Pro } & \multirow{3}{*}{$\tau$} & \multirow{3}{*}{$R$} & \multirow{3}{*}{$a$} & \multirow{3}{*}{$\beta$} & \multicolumn{4}{|c|}{ Branch-and-bound algorithm } & \multirow{2}{*}{\multicolumn{2}{|c|}{$\mathrm{SA}_{1}$}} & \multirow{2}{*}{\multicolumn{2}{|c|}{$\begin{array}{c}\mathrm{SA}_{2} \\
\text { Error percentages }\end{array}$}} & \multirow{2}{*}{\multicolumn{2}{|c|}{$\mathrm{SA}_{3}$}} \\
\hline & & & & & \multicolumn{2}{|c|}{ CPU time } & \multicolumn{2}{|c|}{ Number of nodes } & & & & & & \\
\hline & & & & & Mean & Std & Mean & Std & Mean & Std & Mean & Std & Mean & Std \\
\hline \multirow[t]{48}{*}{0.6} & \multirow[t]{27}{*}{0.40} & \multirow[t]{9}{*}{0.40} & \multirow[t]{3}{*}{1.100} & -1.100 & 169.61 & 485.47 & 5142200 & 14422081 & 0.328 & 1.109 & 0.305 & 0.963 & 0.053 & 0.145 \\
\hline & & & & -1.010 & 139.74 & 443.06 & 4209007 & 13084799 & 0.401 & 0.929 & 0.296 & 0.823 & 0.209 & 0.584 \\
\hline & & & & -1.001 & 141.09 & 445.63 & 4247152 & 13156055 & 0.335 & 0.906 & 0.205 & 0.571 & 0.214 & 0.557 \\
\hline & & & \multirow[t]{3}{*}{1.010} & -1.100 & 154.84 & 442.72 & 4692803 & 13141955 & 0.204 & 0.573 & 0.133 & 0.330 & 0.150 & 0.535 \\
\hline & & & & -1.010 & 128.70 & 407.77 & 3878025 & 12037169 & 0.453 & 1.071 & 0.369 & 0.864 & 0.088 & 0.292 \\
\hline & & & & -1.001 & 129.35 & 404.59 & 3896075 & 11931805 & 0.373 & 1.518 & 0.301 & 0.994 & 0.083 & 0.293 \\
\hline & & & 1.001 & -1.100 & 153.58 & 439.16 & 4655648 & 13037593 & 0.175 & 0.517 & 0.292 & 0.722 & 0.129 & 0.506 \\
\hline & & & & -1.010 & 127.68 & 403.85 & 3847699 & 11919775 & 0.420 & 1.465 & 0.304 & 0.870 & 0.140 & 0.398 \\
\hline & & & & -1.001 & 128.23 & 400.75 & 3863145 & 11818967 & 0.471 & 1.493 & 0.216 & 0.776 & 0.105 & 0.331 \\
\hline & & 0.50 & 1.100 & -1.100 & 148.03 & 468.33 & 4543781 & 14045348 & 0.330 & 0.729 & 0.415 & 0.926 & 0.154 & 0.432 \\
\hline & & & & -1.010 & 135.08 & 466.71 & 4045384 & 13826126 & 0.194 & 0.443 & 0.378 & 1.237 & 0.328 & 1.148 \\
\hline & & & & -1.001 & 134.70 & 467.90 & 4027778 & 13850821 & 0.475 & 1.842 & 0.626 & 2.128 & 0.222 & 1.072 \\
\hline & & & 1.010 & -1.100 & 136.56 & 433.00 & 4186707 & 12960581 & 0.265 & 0.641 & 0.221 & 0.628 & 0.201 & 0.645 \\
\hline & & & & -1.010 & 124.10 & 424.78 & 3717608 & 12569431 & 0.381 & 1.177 & 0.411 & 1.192 & 0.150 & 0.362 \\
\hline & & & & -1.001 & 124.35 & 428.06 & 3721034 & 12662504 & 0.499 & 1.198 & 0.606 & 1.475 & 0.341 & 1.103 \\
\hline & & & 1.001 & -1.100 & 135.22 & 428.57 & 4145141 & 12823831 & 0.288 & 0.749 & 0.464 & 1.740 & 0.069 & 0.163 \\
\hline & & & & -1.010 & 123.87 & 426.05 & 3710684 & 12606768 & 0.386 & 1.203 & 0.473 & 1.274 & 0.267 & 1.089 \\
\hline & & & & -1.001 & 123.62 & 426.51 & 3698366 & 12613179 & 0.576 & 1.492 & 0.522 & 1.329 & 0.237 & 1.065 \\
\hline & & 0.60 & 1.100 & -1.100 & 136.01 & 505.99 & 4109421 & 15175777 & 0.186 & 0.469 & 0.198 & 0.483 & 0.105 & 0.291 \\
\hline & & & & -1.010 & 142.76 & 530.18 & 4297433 & 15833926 & 0.418 & 2.053 & 0.411 & 1.273 & 0.142 & 0.351 \\
\hline & & & & -1.001 & 141.13 & 519.79 & 4243543 & 15506884 & 0.620 & 2.204 & 0.548 & 1.622 & 0.116 & 0.301 \\
\hline & & & 1.010 & -1.100 & 123.90 & 457.10 & 3737374 & 13664018 & 0.560 & 1.963 & 0.200 & 0.679 & 0.119 & 0.422 \\
\hline & & & & -1.010 & 128.19 & 469.11 & 3852210 & 13970930 & 0.596 & 2.184 & 0.464 & 2.182 & 0.070 & 0.175 \\
\hline & & & & -1.001 & 129.10 & 473.67 & 3878835 & 14109979 & 0.334 & 0.897 & 0.498 & 2.114 & 0.148 & 0.360 \\
\hline & & & 1.001 & -1.100 & 123.22 & 455.91 & 3715759 & 13624099 & 0.307 & 0.843 & 0.457 & 1.489 & 0.076 & 0.250 \\
\hline & & & & -1.010 & 126.84 & 463.58 & 3811424 & 13804562 & 0.579 & 2.337 & 0.497 & 1.251 & 0.098 & 0.257 \\
\hline & & & & -1.001 & 127.34 & 466.29 & 3824418 & 13884199 & 0.488 & 2.077 & 0.904 & 3.092 & 0.145 & 0.544 \\
\hline & 0.50 & 0.40 & 1.100 & -1.100 & 50.98 & 137.93 & 1571500 & 4194572 & 0.212 & 0.764 & 0.165 & 0.403 & 0.087 & 0.262 \\
\hline & & & & -1.010 & 44.81 & 132.95 & 1358796 & 3955941 & 0.256 & 0.612 & 0.308 & 0.673 & 0.112 & 0.364 \\
\hline & & & & -1.001 & 44.95 & 133.26 & 1361858 & 3960955 & 0.167 & 0.436 & 0.209 & 0.557 & 0.081 & 0.319 \\
\hline & & & 1.010 & -1.100 & 45.44 & 120.52 & 1403943 & 3668156 & 0.197 & 0.551 & 0.128 & 0.363 & 0.088 & 0.291 \\
\hline & & & & -1.010 & 40.47 & 118.51 & 1230578 & 3534612 & 0.167 & 0.598 & 0.208 & 0.552 & 0.125 & 0.416 \\
\hline & & & & -1.001 & 40.39 & 118.04 & 1226651 & 3514368 & 0.222 & 0.585 & 0.219 & 0.564 & 0.068 & 0.177 \\
\hline & & & 1.001 & -1.100 & 45.04 & 119.11 & 1392216 & 3627488 & 0.093 & 0.189 & 0.309 & 1.317 & 0.079 & 0.280 \\
\hline & & & & -1.010 & 40.14 & 117.44 & 1221284 & 3504993 & 0.310 & 0.829 & 0.217 & 0.619 & 0.115 & 0.450 \\
\hline & & & & -1.001 & 40.01 & 117.02 & 1215980 & 3486325 & 0.189 & 0.480 & 0.218 & 0.567 & 0.101 & 0.292 \\
\hline & & 0.50 & 1.100 & -1.100 & 48.30 & 159.50 & 1462169 & 4805099 & 0.204 & 0.633 & 0.292 & 0.924 & 0.065 & 0.221 \\
\hline & & & & -1.010 & 48.37 & 154.48 & 1462371 & 4586082 & 0.278 & 0.863 & 0.137 & 0.351 & 0.097 & 0.335 \\
\hline & & & & -1.001 & 44.52 & 149.69 & 1363861 & 4460422 & 0.179 & 0.507 & 0.276 & 0.864 & 0.096 & 0.448 \\
\hline & & & 1.010 & -1.100 & 43.14 & 139.72 & 1303877 & 4189668 & 0.307 & 0.779 & 0.301 & 0.844 & 0.111 & 0.471 \\
\hline & & & & -1.010 & 42.79 & 134.24 & 1292152 & 3971867 & 0.407 & 1.195 & 0.461 & 1.175 & 0.096 & 0.345 \\
\hline & & & & -1.001 & 39.34 & 129.88 & 1202979 & 3856027 & 0.324 & 0.762 & 0.298 & 0.903 & 0.130 & 0.489 \\
\hline & & & 1.001 & -1.100 & 42.70 & 138.01 & 1290587 & 4136889 & 0.078 & 0.156 & 0.242 & 0.764 & 0.056 & 0.132 \\
\hline & & & & -1.010 & 42.38 & 133.16 & 1280136 & 3940558 & 0.190 & 0.758 & 0.274 & 0.882 & 0.109 & 0.364 \\
\hline & & & & -1.001 & 38.82 & 127.90 & 1186715 & 3795955 & 0.387 & 1.161 & 0.313 & 1.067 & 0.069 & 0.300 \\
\hline & & 0.60 & 1.100 & -1.100 & 53.09 & 188.20 & 1620698 & 5675353 & 0.208 & 0.528 & 0.317 & 0.941 & 0.091 & 0.424 \\
\hline & & & & -1.010 & 47.40 & 170.15 & 1443635 & 5040348 & 0.151 & 0.434 & 0.214 & 0.490 & 0.052 & 0.134 \\
\hline & & & & -1.001 & 47.20 & 169.46 & 1435927 & 5012344 & 0.244 & 0.482 & 0.155 & 0.441 & 0.068 & 0.206 \\
\hline
\end{tabular}


TABLE 2: Continued.

\begin{tabular}{|c|c|c|c|c|c|c|c|c|c|c|c|c|c|c|}
\hline \multirow{3}{*}{ Pro } & \multirow{3}{*}{$\tau$} & \multirow{3}{*}{$R$} & \multirow{3}{*}{$a$} & \multirow{3}{*}{$\beta$} & \multicolumn{4}{|c|}{ Branch-and-bound algorithm } & \multirow{2}{*}{\multicolumn{2}{|c|}{$\mathrm{SA}_{1}$}} & \multirow{2}{*}{\multicolumn{2}{|c|}{$\begin{array}{c}\mathrm{SA}_{2} \\
\text { Error percentages }\end{array}$}} & \multirow{2}{*}{\multicolumn{2}{|c|}{$\mathrm{SA}_{3}$}} \\
\hline & & & & & \multicolumn{2}{|c|}{ CPU time } & \multicolumn{2}{|c|}{ Number of nodes } & & & & & & \\
\hline & & & & & Mean & Std & Mean & Std & Mean & Std & Mean & Std & Mean & Std \\
\hline & \multirow{33}{*}{0.60} & \multirow{15}{*}{0.40} & \multirow[t]{3}{*}{1.010} & -1.100 & 47.06 & 164.68 & 1431408 & 4933285 & 0.447 & 1.363 & 0.256 & 0.754 & 0.103 & 0.442 \\
\hline & & & & -1.010 & 42.22 & 147.34 & 1282548 & 4342559 & 0.487 & 2.095 & 0.400 & 1.974 & 0.033 & 0.101 \\
\hline & & & & -1.001 & 42.24 & 147.07 & 1282549 & 4331696 & 0.188 & 0.491 & 0.322 & 1.232 & 0.023 & 0.094 \\
\hline & & & \multirow[t]{3}{*}{1.001} & -1.100 & 46.46 & 162.42 & 1412542 & 4862817 & 0.326 & 0.921 & 0.232 & 0.700 & 0.126 & 0.430 \\
\hline & & & & -1.010 & 41.82 & 145.44 & 1270995 & 4287523 & 0.392 & 1.066 & 0.129 & 0.371 & 0.023 & 0.073 \\
\hline & & & & -1.001 & 41.70 & 144.93 & 1265860 & 4267324 & 0.278 & 1.021 & 0.202 & 0.757 & 0.047 & 0.125 \\
\hline & & & \multirow[t]{3}{*}{1.100} & -1.100 & 9.52 & 22.00 & 301712 & 689889 & 0.159 & 0.491 & 0.262 & 0.958 & 0.218 & 0.871 \\
\hline & & & & -1.010 & 9.40 & 23.76 & 300052 & 754248 & 0.107 & 0.299 & 0.111 & 0.365 & 0.095 & 0.330 \\
\hline & & & & -1.001 & 9.93 & 24.06 & 319053 & 765992 & 0.218 & 0.955 & 0.133 & 0.494 & 0.030 & 0.093 \\
\hline & & & \multirow[t]{3}{*}{1.010} & -1.100 & 8.54 & 19.68 & 272224 & 621655 & 0.247 & 0.766 & 0.198 & 0.718 & 0.152 & 0.686 \\
\hline & & & & -1.010 & 8.34 & 20.87 & 267808 & 667802 & 0.350 & 1.659 & 0.072 & 0.193 & 0.049 & 0.126 \\
\hline & & & & -1.001 & 8.81 & 21.16 & 284571 & 678948 & 0.268 & 0.826 & 0.086 & 0.207 & 0.041 & 0.094 \\
\hline & & & \multirow[t]{3}{*}{1.001} & -1.100 & 8.43 & 19.43 & 268841 & 613948 & 0.105 & 0.290 & 0.229 & 0.704 & 0.045 & 0.117 \\
\hline & & & & -1.010 & 8.24 & 20.59 & 264663 & 659399 & 0.325 & 1.427 & 0.292 & 1.349 & 0.038 & 0.103 \\
\hline & & & & -1.001 & 8.72 & 20.89 & 281716 & 670765 & 0.286 & 1.191 & 0.198 & 0.592 & 0.065 & 0.144 \\
\hline & & \multirow[t]{9}{*}{0.50} & \multirow[t]{3}{*}{1.100} & -1.100 & 12.59 & 32.85 & 402441 & 1039572 & 0.080 & 0.185 & 0.061 & 0.191 & 0.040 & 0.136 \\
\hline & & & & -1.010 & 13.08 & 34.50 & 415790 & 1088849 & 0.149 & 0.413 & 0.102 & 0.220 & 0.064 & 0.188 \\
\hline & & & & -1.001 & 13.20 & 34.77 & 419385 & 1097323 & 0.115 & 0.350 & 0.211 & 0.597 & 0.064 & 0.213 \\
\hline & & & \multirow[t]{3}{*}{1.010} & -1.100 & 11.23 & 29.11 & 360464 & 926431 & 0.185 & 0.681 & 0.037 & 0.095 & 0.032 & 0.071 \\
\hline & & & & -1.010 & 11.52 & 30.03 & 367316 & 953452 & 0.081 & 0.260 & 0.256 & 0.747 & 0.057 & 0.240 \\
\hline & & & & -1.001 & 11.57 & 30.13 & 368553 & 956769 & 0.178 & 0.488 & 0.098 & 0.226 & 0.106 & 0.346 \\
\hline & & & \multirow[t]{3}{*}{1.001} & -1.100 & 11.13 & 28.89 & 357394 & 919613 & 0.115 & 0.411 & 0.272 & 1.049 & 0.042 & 0.177 \\
\hline & & & & -1.010 & 11.36 & 29.59 & 362362 & 939892 & 0.207 & 0.593 & 0.129 & 0.379 & 0.062 & 0.256 \\
\hline & & & & -1.001 & 11.42 & 29.70 & 364005 & 943583 & 0.108 & 0.337 & 0.186 & 0.628 & 0.060 & 0.249 \\
\hline & & \multirow[t]{9}{*}{0.60} & 1.100 & -1.100 & 17.00 & 47.56 & 540126 & 1496760 & 0.207 & 0.619 & 0.101 & 0.249 & 0.079 & 0.281 \\
\hline & & & & -1.010 & 16.32 & 47.42 & 511056 & 1470526 & 0.229 & 0.549 & 0.122 & 0.380 & 0.056 & 0.219 \\
\hline & & & & -1.001 & 16.26 & 47.28 & 508286 & 1462393 & 0.177 & 0.495 & 0.135 & 0.339 & 0.088 & 0.251 \\
\hline & & & 1.010 & -1.100 & 15.27 & 42.24 & 485259 & 1329067 & 0.197 & 0.635 & 0.176 & 0.465 & 0.083 & 0.387 \\
\hline & & & & -1.010 & 14.66 & 41.81 & 460159 & 1302846 & 0.195 & 0.545 & 0.164 & 0.434 & 0.075 & 0.213 \\
\hline & & & & -1.001 & 14.51 & 41.24 & 453999 & 1279281 & 0.184 & 0.493 & 0.112 & 0.331 & 0.137 & 0.409 \\
\hline & & & 1.001 & -1.100 & 15.14 & 41.77 & 481166 & 1314352 & 0.118 & 0.279 & 0.301 & 0.800 & 0.050 & 0.136 \\
\hline & & & & -1.010 & 14.47 & 41.18 & 453836 & 1283057 & 0.160 & 0.487 & 0.204 & 0.512 & 0.049 & 0.155 \\
\hline & & & & -1.001 & 14.33 & 40.61 & 448251 & 1259365 & 0.122 & 0.411 & 0.294 & 0.807 & 0.098 & 0.356 \\
\hline & Averag & & & & 63.62 & 208.26 & 1929543 & 6212496 & 0.277 & 0.870 & 0.272 & 0.841 & 0.104 & 0.352 \\
\hline
\end{tabular}

in the weighted shortest processing time (WSPT) order. In order to get a good initial solution, we apply the NEH method [31] to the initial sequences produced by $\mathrm{SA}_{2}$ and $\mathrm{SA}_{3}$.

Step 2. Neighborhood generation.

Neighborhood generation plays an important role in the efficiency of SA algorithms. We use the pairwise interchange (PI) neighborhood generation method in the algorithms.

Step 3. Acceptance probability.

When a new feasible sequence is generated, it is accepted if its objective value is smaller than that of the original sequence; otherwise, it is accepted with a probability that decreases as the process evolves. The probability of acceptance is generated from an exponential distribution as follows:

$$
P(\text { accept })=\exp (-\rho \times \Delta W T C)
$$

where $\rho$ is a control parameter and $\triangle W T C$ is the change in the objective value. In addition, we use the method suggested by Ben-Arieh and Maimon [32] to change $\rho$ in the $k$ th iteration as follows:

$$
\rho=\frac{k}{\delta}
$$

where $\delta$ is an experimental constant. After preliminary trials, we used $\delta=0.8$ in our experiments.

If the total weighted completion time increases as a result of a random pairwise interchange, the new sequence is accepted when $P$ (accept) $>r$, where $r$ is randomly sampled from the uniform distribution $U(0,1)$.

Step 4. Stopping condition.

Our preliminary trials indicate that the quality of the schedule is stable after $200 n$ iterations, where $n$ is the number of jobs. 
TABLE 3: Performance of the branch-and-bound and SA algorithms $(n=15$, pro $=0.4)$.

\begin{tabular}{|c|c|c|c|c|c|c|c|c|c|c|c|c|c|c|}
\hline \multirow{3}{*}{ Pro } & \multirow{3}{*}{$\tau$} & \multirow{3}{*}{$R$} & \multirow{3}{*}{$a$} & \multirow{3}{*}{$\beta$} & \multicolumn{4}{|c|}{ Branch-and-bound algorithm } & \multirow{2}{*}{\multicolumn{2}{|c|}{$\mathrm{SA}_{1}$}} & \multirow{2}{*}{\multicolumn{2}{|c|}{$\begin{array}{c}\mathrm{SA}_{2} \\
\text { Error percentages }\end{array}$}} & \multirow{2}{*}{\multicolumn{2}{|c|}{$\mathrm{SA}_{3}$}} \\
\hline & & & & & \multicolumn{2}{|c|}{ CPU time } & \multicolumn{2}{|c|}{ Number of nodes } & & & & & & \\
\hline & & & & & Mean & Std & Mean & Std & Mean & Std & Mean & Std & Mean & Std \\
\hline \multirow[t]{48}{*}{0.4} & \multirow[t]{27}{*}{0.40} & \multirow[t]{9}{*}{0.40} & \multirow[t]{3}{*}{1.100} & -1.100 & 311.88 & 414.22 & 11758285 & 15903115 & 0.837 & 1.546 & 0.887 & 1.652 & 0.293 & 0.483 \\
\hline & & & & -1.010 & 191.71 & 349.53 & 7136396 & 13128691 & 0.456 & 0.679 & 0.745 & 1.130 & 0.541 & 1.062 \\
\hline & & & & -1.001 & 196.36 & 358.32 & 7305845 & 13462940 & 0.519 & 0.683 & 0.491 & 0.745 & 0.397 & 0.537 \\
\hline & & & \multirow[t]{3}{*}{1.010} & -1.100 & 287.40 & 379.73 & 10866955 & 14623008 & 0.321 & 0.367 & 0.821 & 1.332 & 0.279 & 0.467 \\
\hline & & & & -1.010 & 175.58 & 319.29 & 6557095 & 12024063 & 0.543 & 0.917 & 0.347 & 0.504 & 0.443 & 0.911 \\
\hline & & & & -1.001 & 178.59 & 324.07 & 6665084 & 12209228 & 0.497 & 0.661 & 0.915 & 1.382 & 0.359 & 0.655 \\
\hline & & & 1.001 & -1.100 & 285.39 & 376.85 & 10796044 & 14522042 & 0.855 & 1.659 & 1.354 & 2.175 & 0.220 & 0.281 \\
\hline & & & & -1.010 & 174.07 & 316.36 & 6503448 & 11918702 & 0.401 & 0.694 & 1.049 & 1.572 & 0.379 & 0.764 \\
\hline & & & & -1.001 & 177.12 & 321.43 & 6613663 & 12115285 & 0.320 & 0.576 & 0.313 & 0.531 & 0.401 & 0.804 \\
\hline & & 0.50 & 1.100 & -1.100 & 257.34 & 384.96 & 9612742 & 14644726 & 1.302 & 2.391 & 0.531 & 0.862 & 0.474 & 0.639 \\
\hline & & & & -1.010 & 206.07 & 398.32 & 7644147 & 15064816 & 0.744 & 1.464 & 0.588 & 1.103 & 0.267 & 0.370 \\
\hline & & & & -1.001 & 205.08 & 404.74 & 7648212 & 15313361 & 1.871 & 3.131 & 0.438 & 0.558 & 0.433 & 0.707 \\
\hline & & & 1.010 & -1.100 & 239.89 & 359.34 & 8982129 & 13695175 & 1.282 & 1.814 & 0.821 & 1.005 & 0.600 & 1.058 \\
\hline & & & & -1.010 & 189.79 & 368.05 & 7057756 & 13951685 & 1.599 & 2.800 & 1.104 & 2.457 & 0.612 & 1.004 \\
\hline & & & & -1.001 & 187.82 & 370.71 & 7020711 & 14059100 & 0.580 & 1.139 & 0.729 & 1.177 & 0.305 & 0.446 \\
\hline & & & 1.001 & -1.100 & 237.98 & 356.54 & 8913945 & 13593996 & 0.803 & 1.032 & 1.179 & 1.838 & 0.451 & 0.742 \\
\hline & & & & -1.010 & 188.22 & 365.16 & 7000983 & 13842236 & 0.633 & 1.160 & 1.384 & 2.292 & 0.945 & 2.301 \\
\hline & & & & -1.001 & 186.16 & 367.78 & 6960609 & 13950622 & 0.999 & 1.431 & 0.214 & 0.254 & 0.236 & 0.457 \\
\hline & & 0.60 & 1.100 & -1.100 & 226.20 & 413.41 & 8376937 & 15502069 & 0.799 & 1.222 & 0.921 & 1.162 & 0.507 & 0.894 \\
\hline & & & & -1.010 & 193.13 & 404.47 & 7149216 & 15170536 & 1.688 & 1.715 & 1.225 & 1.728 & 0.636 & 0.847 \\
\hline & & & & -1.001 & 196.52 & 409.14 & 7277271 & 15334414 & 0.781 & 1.150 & 0.665 & 1.036 & 0.736 & 0.988 \\
\hline & & & 1.010 & -1.100 & 213.76 & 391.38 & 7940293 & 14704311 & 1.473 & 2.165 & 0.742 & 1.324 & 0.371 & 0.619 \\
\hline & & & & -1.010 & 179.59 & 377.57 & 6671217 & 14223196 & 0.831 & 0.898 & 1.027 & 1.720 & 0.526 & 0.744 \\
\hline & & & & -1.001 & 182.23 & 380.25 & 6770069 & 14300776 & 1.536 & 2.013 & 0.802 & 1.033 & 0.511 & 0.739 \\
\hline & & & 1.001 & -1.100 & 212.06 & 388.42 & 7877739 & 14594634 & 0.999 & 1.440 & 0.665 & 1.477 & 0.328 & 0.477 \\
\hline & & & & -1.010 & 180.39 & 378.71 & 6706222 & 14252125 & 1.393 & 1.856 & 0.981 & 1.287 & 0.632 & 0.764 \\
\hline & & & & -1.001 & 180.54 & 376.59 & 6707971 & 14165604 & 0.962 & 1.708 & 1.115 & 2.115 & 0.580 & 0.853 \\
\hline & 0.50 & 0.40 & 1.100 & -1.100 & 59.79 & 92.20 & 2340463 & 3674295 & 0.742 & 1.792 & 0.599 & 1.025 & 0.334 & 0.513 \\
\hline & & & & -1.010 & 90.67 & 113.76 & 3549507 & 4515071 & 0.599 & 0.900 & 0.461 & 0.727 & 0.264 & 0.388 \\
\hline & & & & -1.001 & 92.71 & 115.83 & 3626112 & 4594477 & 0.440 & 0.675 & 0.393 & 0.663 & 0.261 & 0.351 \\
\hline & & & 1.010 & -1.100 & 54.94 & 84.53 & 2162741 & 3386252 & 0.581 & 0.882 & 0.424 & 0.726 & 0.187 & 0.339 \\
\hline & & & & -1.010 & 82.68 & 104.10 & 3254738 & 4152699 & 0.479 & 0.703 & 0.728 & 1.174 & 0.235 & 0.437 \\
\hline & & & & -1.001 & 84.48 & 105.83 & 3322651 & 4219459 & 2.067 & 5.768 & 0.738 & 1.153 & 0.142 & 0.179 \\
\hline & & & 1.001 & -1.100 & 54.44 & 83.79 & 2144562 & 3358398 & 0.196 & 0.280 & 0.575 & 0.964 & 0.274 & 0.430 \\
\hline & & & & -1.010 & 81.88 & 103.15 & 3224787 & 4117010 & 0.760 & 1.991 & 0.586 & 1.682 & 0.138 & 0.224 \\
\hline & & & & -1.001 & 83.65 & 104.87 & 3291685 & 4183898 & 0.281 & 0.436 & 0.496 & 0.936 & 0.177 & 0.290 \\
\hline & & 0.50 & 1.100 & -1.100 & 83.54 & 109.89 & 3264865 & 4371726 & 0.624 & 0.706 & 0.660 & 0.789 & 0.350 & 0.522 \\
\hline & & & & -1.010 & 82.99 & 112.28 & 3200949 & 4429808 & 1.369 & 2.181 & 0.660 & 1.275 & 0.215 & 0.348 \\
\hline & & & & -1.001 & 83.65 & 115.22 & 3232620 & 4539750 & 1.125 & 1.877 & 1.135 & 2.213 & 0.153 & 0.205 \\
\hline & & & 1.010 & -1.100 & 76.35 & 100.18 & 2999791 & 3999506 & 0.423 & 0.698 & 0.837 & 1.304 & 0.240 & 0.269 \\
\hline & & & & -1.010 & 76.27 & 102.66 & 2954127 & 4065085 & 1.511 & 2.811 & 1.110 & 1.802 & 0.416 & 1.179 \\
\hline & & & & -1.001 & 77.07 & 105.75 & 2990752 & 4183939 & 0.973 & 1.764 & 1.021 & 1.797 & 0.412 & 1.167 \\
\hline & & & 1.001 & -1.100 & 75.65 & 99.35 & 2974524 & 3968512 & 1.006 & 1.300 & 0.245 & 0.309 & 0.346 & 0.479 \\
\hline & & & & -1.010 & 75.59 & 101.72 & 2929144 & 4029183 & 1.105 & 1.705 & 0.672 & 1.040 & 0.745 & 1.588 \\
\hline & & & & -1.001 & 76.26 & 104.60 & 2960124 & 4138909 & 0.786 & 1.267 & 0.804 & 1.519 & 0.442 & 1.168 \\
\hline & & 0.60 & 1.100 & -1.100 & 97.45 & 122.49 & 3776266 & 4802973 & 1.069 & 1.723 & 0.649 & 0.835 & 0.273 & 0.393 \\
\hline & & & & -1.010 & 67.32 & 119.31 & 2539341 & 4508240 & 1.642 & 2.544 & 1.020 & 1.764 & 0.243 & 0.349 \\
\hline & & & & -1.001 & 68.51 & 121.41 & 2582654 & 4586272 & 0.708 & 0.920 & 1.773 & 2.473 & 0.406 & 0.452 \\
\hline
\end{tabular}


TABLe 3: Continued.

\begin{tabular}{|c|c|c|c|c|c|c|c|c|c|c|c|c|c|c|}
\hline \multirow{3}{*}{ Pro } & \multirow{3}{*}{$\tau$} & \multirow{3}{*}{$R$} & \multirow{3}{*}{$a$} & \multirow{3}{*}{$\beta$} & \multicolumn{4}{|c|}{ Branch-and-bound algorithm } & \multirow{2}{*}{\multicolumn{2}{|c|}{$\mathrm{SA}_{1}$}} & \multirow{2}{*}{\multicolumn{2}{|c|}{$\begin{array}{c}\mathrm{SA}_{2} \\
\text { Error percentages }\end{array}$}} & \multicolumn{2}{|c|}{$\mathrm{SA}_{3}$} \\
\hline & & & & & \multicolumn{2}{|c|}{ CPU time } & \multicolumn{2}{|c|}{ Number of nodes } & & & & & \multirow[b]{2}{*}{ Mean } & \multirow[b]{2}{*}{ Std } \\
\hline & & & & & Mean & Std & Mean & Std & Mean & Std & \multicolumn{2}{|c|}{$\begin{array}{c}\text { Error percentages } \\
\text { Mean Std }\end{array}$} & & \\
\hline & \multirow{33}{*}{0.60} & \multirow{15}{*}{0.40} & \multirow[t]{3}{*}{1.010} & -1.100 & 89.87 & 112.61 & 3489778 & 4415326 & 1.060 & 1.675 & 0.695 & 1.240 & 0.286 & 0.411 \\
\hline & & & & -1.010 & 63.04 & 112.01 & 2390213 & 4253672 & 1.072 & 1.535 & 1.014 & 1.487 & 0.604 & 1.236 \\
\hline & & & & -1.001 & 63.99 & 113.55 & 2423954 & 4309165 & 1.790 & 3.392 & 1.329 & 2.338 & 0.405 & 0.753 \\
\hline & & & \multirow[t]{3}{*}{1.001} & -1.100 & 89.25 & 111.59 & 3467072 & 4375682 & 0.662 & 1.008 & 0.744 & 0.983 & 0.361 & 0.630 \\
\hline & & & & -1.010 & 62.73 & 111.02 & 2379099 & 4216867 & 1.321 & 1.547 & 1.916 & 2.870 & 0.545 & 1.139 \\
\hline & & & & -1.001 & 63.45 & 112.61 & 2404690 & 4275428 & 1.255 & 1.815 & 1.098 & 1.540 & 0.690 & 1.367 \\
\hline & & & \multirow[t]{3}{*}{1.100} & -1.100 & 5.85 & 7.71 & 236881 & 324192 & 0.296 & 0.468 & 0.224 & 0.323 & 0.283 & 0.505 \\
\hline & & & & -1.010 & 5.16 & 9.04 & 209445 & 379285 & 0.329 & 0.464 & 0.291 & 0.539 & 0.175 & 0.331 \\
\hline & & & & -1.001 & 5.16 & 9.12 & 210367 & 382778 & 0.384 & 0.530 & 0.403 & 0.553 & 0.231 & 0.498 \\
\hline & & & \multirow[t]{3}{*}{1.010} & -1.100 & 5.44 & 7.26 & 221007 & 305841 & 0.295 & 0.405 & 0.146 & 0.226 & 0.289 & 0.553 \\
\hline & & & & -1.010 & 4.68 & 8.14 & 190945 & 342880 & 0.763 & 2.524 & 0.354 & 0.540 & 0.189 & 0.295 \\
\hline & & & & -1.001 & 4.66 & 8.23 & 190746 & 346282 & 0.312 & 0.426 & 0.328 & 0.502 & 0.232 & 0.440 \\
\hline & & & \multirow[t]{3}{*}{1.001} & -1.100 & 5.40 & 7.24 & 219717 & 305198 & 0.392 & 0.510 & 0.299 & 0.354 & 0.173 & 0.306 \\
\hline & & & & -1.010 & 4.64 & 8.06 & 189163 & 339587 & 0.361 & 0.627 & 0.360 & 0.618 & 0.226 & 0.397 \\
\hline & & & & -1.001 & 4.62 & 8.16 & 189126 & 343494 & 0.928 & 2.476 & 0.467 & 0.805 & 0.113 & 0.241 \\
\hline & & 0.50 & 1.100 & -1.100 & 6.94 & 11.68 & 284387 & 494376 & 0.477 & 0.920 & 0.423 & 0.860 & 0.206 & 0.347 \\
\hline & & & & -1.010 & 9.15 & 14.39 & 373361 & 598325 & 0.587 & 1.147 & 0.589 & 1.004 & 0.402 & 1.021 \\
\hline & & & & -1.001 & 9.32 & 14.58 & 380130 & 606005 & 1.007 & 1.916 & 0.949 & 1.703 & 0.257 & 0.463 \\
\hline & & & 1.010 & -1.100 & 6.31 & 10.69 & 259327 & 453058 & 0.502 & 0.637 & 0.389 & 0.784 & 0.228 & 0.344 \\
\hline & & & & -1.010 & 8.33 & 13.23 & 341436 & 552785 & 1.149 & 1.823 & 0.394 & 0.628 & 0.139 & 0.191 \\
\hline & & & & -1.001 & 8.49 & 13.40 & 347732 & 559407 & 0.687 & 1.390 & 0.730 & 1.267 & 0.150 & 0.291 \\
\hline & & & 1.001 & -1.100 & 6.25 & 10.59 & 256783 & 448847 & 0.621 & 1.179 & 0.247 & 0.368 & 0.183 & 0.337 \\
\hline & & & & -1.010 & 8.24 & 13.11 & 338060 & 547960 & 0.329 & 0.561 & 0.680 & 1.277 & 0.112 & 0.182 \\
\hline & & & & -1.001 & 8.40 & 13.28 & 344399 & 554855 & 0.445 & 1.069 & 1.128 & 2.085 & 0.151 & 0.324 \\
\hline & & 0.60 & 1.100 & -1.100 & 10.29 & 16.81 & 417314 & 689695 & 0.410 & 0.900 & 0.796 & 1.484 & 0.174 & 0.333 \\
\hline & & & & -1.010 & 14.72 & 19.92 & 606522 & 827274 & 0.225 & 0.496 & 0.303 & 0.348 & 0.179 & 0.276 \\
\hline & & & & -1.001 & 15.05 & 20.21 & 620128 & 838524 & 0.965 & 2.173 & 0.872 & 2.173 & 0.238 & 0.361 \\
\hline & & & 1.010 & -1.100 & 9.34 & 15.30 & 380584 & 630421 & 0.599 & 1.508 & 0.636 & 1.551 & 0.267 & 0.519 \\
\hline & & & & -1.010 & 13.41 & 18.26 & 554783 & 760674 & 0.865 & 2.444 & 0.231 & 0.319 & 0.141 & 0.308 \\
\hline & & & & -1.001 & 13.70 & 18.52 & 566713 & 770556 & 1.279 & 3.789 & 1.013 & 3.830 & 0.111 & 0.210 \\
\hline & & & 1.001 & -1.100 & 9.26 & 15.17 & 377334 & 625018 & 0.509 & 1.005 & 0.630 & 0.744 & 0.282 & 0.528 \\
\hline & & & & -1.010 & 13.29 & 18.10 & 550084 & 754492 & 1.052 & 2.524 & 0.591 & 0.707 & 0.104 & 0.132 \\
\hline & & & & -1.001 & 13.58 & 18.37 & 561776 & 764270 & 0.919 & 2.456 & 1.241 & 3.799 & 0.261 & 0.455 \\
\hline & Avera & & & & 97.89 & 164.20 & 3701438 & 6277631 & 0.832 & 1.464 & 0.730 & 1.253 & 0.335 & 0.588 \\
\hline
\end{tabular}

3.4. Details of the Branch-and-Bound Algorithm. We use the depth-first search in the branching procedure and assign jobs in a forward manner starting with the first position. In the searching tree, we adopt a branch and systematically work down the tree until we either eliminate it by virtue of the dominant properties and the lower bounds or reach its final node, in which case this sequence either replaces the initial solution or is eliminated. We summarize the main steps in the following.

\subsubsection{The Branch-and-Bound Algorithm}

Step 1. Apply the best solution obtained from the three proposed simulated annealing algorithms as the initial solution for the branch-and-bound algorithm.

Step 2. Use the dominant rules 1-5 to eliminate the dominated partial sequences.
Step 3. Compute the lower bound of the total weighted completion time of the first agent for the unscheduled partial sequences or the total weighted completion time of the first agent for the completed sequences. If the lower bound for an unscheduled partial sequence is greater than that of the initial solution, eliminate that node and all the nodes beyond it in the branch. If the value of the completed sequence is less than that of the initial solution, replace it as the new solution. Otherwise, eliminate it.

Step 4. Repeat Steps 2 and 3 until all the branches are explored.

\section{Computational Experiments}

We conducted extensive computational experiments to evaluate the efficiency of the branch-and-bound algorithm and the performance of the three simulated annealing algorithms. 
TABLE 4: Performance of the branch-and-bound and SA algorithms $(n=15$, pro $=0.6)$.

\begin{tabular}{|c|c|c|c|c|c|c|c|c|c|c|c|c|c|c|}
\hline \multirow{3}{*}{ Pro } & \multirow{3}{*}{$\tau$} & \multirow{3}{*}{$R$} & \multirow{3}{*}{$a$} & \multirow{3}{*}{$\beta$} & \multicolumn{4}{|c|}{ Branch-and-bound algorithm } & \multirow{2}{*}{\multicolumn{2}{|c|}{$\mathrm{SA}_{1}$}} & \multirow{2}{*}{\multicolumn{2}{|c|}{$\begin{array}{c}\mathrm{SA}_{2} \\
\text { Error percentages }\end{array}$}} & \multirow{2}{*}{\multicolumn{2}{|c|}{$\mathrm{SA}_{3}$}} \\
\hline & & & & & \multicolumn{2}{|c|}{ CPU time } & \multicolumn{2}{|c|}{ Number of nodes } & & & & & & \\
\hline & & & & & Mean & Std & Mean & Std & Mean & Std & Mean & Std & Mean & Std \\
\hline \multirow[t]{48}{*}{0.6} & \multirow[t]{27}{*}{0.40} & \multirow[t]{9}{*}{0.40} & \multirow[t]{3}{*}{1.100} & -1.100 & 508.76 & 747.32 & 15421452 & 22053238 & 0.962 & 1.783 & 0.916 & 1.513 & 0.151 & 0.221 \\
\hline & & & & -1.010 & 419.13 & 702.28 & 12621515 & 20637904 & 0.944 & 1.289 & 0.655 & 1.047 & 0.516 & 0.838 \\
\hline & & & & -1.001 & 423.19 & 705.68 & 12735894 & 20730667 & 0.864 & 1.318 & 0.479 & 0.725 & 0.453 & 0.721 \\
\hline & & & \multirow[t]{3}{*}{1.010} & -1.100 & 464.44 & 681.30 & 14073410 & 20085625 & 0.556 & 0.892 & 0.396 & 0.477 & 0.421 & 0.871 \\
\hline & & & & -1.010 & 386.03 & 646.22 & 11628829 & 18977908 & 1.008 & 1.105 & 0.885 & 1.240 & 0.158 & 0.233 \\
\hline & & & & -1.001 & 387.96 & 639.10 & 11682914 & 18744229 & 1.092 & 2.516 & 0.810 & 1.574 & 0.248 & 0.474 \\
\hline & & & 1.001 & -1.100 & 460.68 & 675.83 & 13961961 & 19925977 & 0.459 & 0.816 & 0.786 & 1.068 & 0.329 & 0.833 \\
\hline & & & & -1.010 & 382.96 & 639.75 & 11537878 & 18783161 & 1.160 & 2.383 & 0.759 & 1.344 & 0.288 & 0.527 \\
\hline & & & & -1.001 & 384.61 & 632.89 & 11584157 & 18561838 & 1.290 & 2.378 & 0.538 & 1.226 & 0.232 & 0.481 \\
\hline & & 0.50 & 1.100 & -1.100 & 444.01 & 741.94 & 13626115 & 22119158 & 0.878 & 1.042 & 0.949 & 1.204 & 0.399 & 0.667 \\
\hline & & & & -1.010 & 405.16 & 754.23 & 12130822 & 22295569 & 0.458 & 0.568 & 0.486 & 0.672 & 0.241 & 0.277 \\
\hline & & & & -1.001 & 404.01 & 756.97 & 12077970 & 22363737 & 0.864 & 2.460 & 1.275 & 3.130 & 0.218 & 0.328 \\
\hline & & & 1.010 & -1.100 & 409.60 & 686.34 & 12555127 & 20418894 & 0.713 & 0.937 & 0.648 & 0.968 & 0.600 & 1.020 \\
\hline & & & & -1.010 & 372.23 & 685.16 & 11147650 & 20223236 & 0.667 & 0.883 & 0.429 & 0.645 & 0.336 & 0.431 \\
\hline & & & & -1.001 & 372.98 & 691.25 & 11157930 & 20400814 & 0.846 & 0.881 & 0.988 & 1.254 & 0.420 & 0.469 \\
\hline & & & 1.001 & -1.100 & 405.58 & 679.26 & 12430454 & 20200090 & 0.845 & 1.118 & 0.705 & 0.787 & 0.163 & 0.225 \\
\hline & & & & -1.010 & 371.52 & 687.89 & 11126894 & 20304074 & 0.743 & 0.970 & 0.796 & 1.162 & 0.222 & 0.314 \\
\hline & & & & -1.001 & 370.77 & 689.06 & 11089959 & 20330734 & 1.036 & 1.723 & 0.844 & 0.984 & 0.216 & 0.228 \\
\hline & & 0.60 & 1.100 & -1.100 & 407.95 & 829.01 & 12323340 & 24833584 & 0.477 & 0.681 & 0.558 & 0.713 & 0.173 & 0.274 \\
\hline & & & & -1.010 & 428.20 & 868.38 & 12887492 & 25900159 & 0.322 & 0.471 & 0.732 & 1.121 & 0.338 & 0.491 \\
\hline & & & & -1.001 & 423.31 & 850.13 & 12725974 & 25328476 & 0.944 & 0.908 & 0.493 & 0.828 & 0.324 & 0.451 \\
\hline & & & 1.010 & -1.100 & 371.63 & 747.82 & 11207421 & 22320678 & 1.601 & 3.184 & 0.469 & 1.084 & 0.147 & 0.271 \\
\hline & & & & -1.010 & 384.51 & 766.39 & 11551885 & 22789194 & 0.784 & 1.413 & 0.410 & 0.668 & 0.166 & 0.236 \\
\hline & & & & -1.001 & 387.24 & 774.19 & 11631830 & 23028425 & 0.788 & 1.386 & 0.514 & 0.841 & 0.168 & 0.240 \\
\hline & & & 1.001 & -1.100 & 369.58 & 746.26 & 11142589 & 22266752 & 0.446 & 0.570 & 1.112 & 2.195 & 0.229 & 0.396 \\
\hline & & & & -1.010 & 380.45 & 757.19 & 11429538 & 22512411 & 0.489 & 0.591 & 1.124 & 1.760 & 0.230 & 0.329 \\
\hline & & & & -1.001 & 381.94 & 761.87 & 11468577 & 22651834 & 0.633 & 0.831 & 0.521 & 0.692 & 0.152 & 0.236 \\
\hline & 0.50 & 0.40 & 1.100 & -1.100 & 152.89 & 208.18 & 4712381 & 6298903 & 0.599 & 1.252 & 0.439 & 0.590 & 0.242 & 0.411 \\
\hline & & & & -1.010 & 134.40 & 206.96 & 4074096 & 6124008 & 0.559 & 0.843 & 0.669 & 0.919 & 0.311 & 0.582 \\
\hline & & & & -1.001 & 134.81 & 207.42 & 4083266 & 6129901 & 0.365 & 0.507 & 0.521 & 0.845 & 0.237 & 0.527 \\
\hline & & & 1.010 & -1.100 & 136.30 & 180.49 & 4209770 & 5461139 & 0.520 & 0.850 & 0.375 & 0.559 & 0.257 & 0.466 \\
\hline & & & & -1.010 & 121.37 & 183.77 & 3689527 & 5448570 & 0.501 & 0.968 & 0.576 & 0.846 & 0.374 & 0.664 \\
\hline & & & & -1.001 & 121.14 & 182.92 & 3677737 & 5412926 & 0.470 & 0.787 & 0.590 & 0.844 & 0.163 & 0.257 \\
\hline & & & 1.001 & -1.100 & 135.09 & 178.19 & 4174596 & 5394367 & 0.224 & 0.239 & 0.406 & 0.611 & 0.232 & 0.453 \\
\hline & & & & -1.010 & 120.38 & 182.07 & 3661654 & 5401469 & 0.899 & 1.257 & 0.604 & 0.969 & 0.315 & 0.747 \\
\hline & & & & -1.001 & 120.00 & 181.39 & 3645728 & 5371004 & 0.536 & 0.712 & 0.585 & 0.862 & 0.280 & 0.460 \\
\hline & & 0.50 & 1.100 & -1.100 & 144.86 & 255.28 & 4384365 & 7682740 & 0.587 & 1.003 & 0.817 & 1.479 & 0.184 & 0.358 \\
\hline & & & & -1.010 & 145.08 & 245.30 & 4385018 & 7249908 & 0.727 & 1.340 & 0.297 & 0.467 & 0.218 & 0.521 \\
\hline & & & & -1.001 & 133.52 & 240.54 & 4089473 & 7123797 & 0.477 & 0.789 & 0.784 & 1.369 & 0.274 & 0.754 \\
\hline & & & 1.010 & -1.100 & 129.39 & 222.62 & 3909542 & 6663581 & 0.724 & 1.174 & 0.855 & 1.298 & 0.331 & 0.781 \\
\hline & & & & -1.010 & 128.34 & 212.22 & 3874419 & 6246018 & 1.114 & 1.876 & 1.283 & 1.748 & 0.198 & 0.435 \\
\hline & & & & -1.001 & 118.00 & 207.86 & 3606887 & 6129381 & 0.704 & 0.987 & 0.822 & 1.424 & 0.226 & 0.476 \\
\hline & & & 1.001 & -1.100 & 128.07 & 219.79 & 3869681 & 6575772 & 0.215 & 0.208 & 0.614 & 1.188 & 0.160 & 0.190 \\
\hline & & & & -1.010 & 127.12 & 210.58 & 3838378 & 6199175 & 0.548 & 1.256 & 0.731 & 1.386 & 0.239 & 0.472 \\
\hline & & & & -1.001 & 116.43 & 204.61 & 3558106 & 6030835 & 1.064 & 1.816 & 0.919 & 1.717 & 0.111 & 0.338 \\
\hline & & 0.60 & 1.100 & -1.100 & 159.25 & 305.68 & 4859947 & 9196852 & 0.533 & 0.741 & 0.935 & 1.465 & 0.258 & 0.714 \\
\hline & & & & -1.010 & 142.16 & 277.02 & 4328607 & 8163288 & 0.417 & 0.676 & 0.571 & 0.712 & 0.138 & 0.199 \\
\hline & & & & -1.001 & 141.57 & 275.89 & 4305544 & 8117557 & 0.724 & 0.597 & 0.381 & 0.678 & 0.202 & 0.322 \\
\hline
\end{tabular}


TABLE 4: Continued.

\begin{tabular}{|c|c|c|c|c|c|c|c|c|c|c|c|c|c|c|}
\hline \multirow{3}{*}{ Pro } & \multirow{3}{*}{$\tau$} & \multirow{3}{*}{$R$} & \multirow{3}{*}{$a$} & \multirow{3}{*}{$\beta$} & \multicolumn{4}{|c|}{ Branch-and-bound algorithm } & \multirow{2}{*}{\multicolumn{2}{|c|}{$\mathrm{SA}_{1}$}} & \multirow{2}{*}{\multicolumn{2}{|c|}{$\begin{array}{c}\mathrm{SA}_{2} \\
\text { Error percentages }\end{array}$}} & \multicolumn{2}{|c|}{$\mathrm{SA}_{3}$} \\
\hline & & & & & \multicolumn{2}{|c|}{ CPU time } & \multicolumn{2}{|c|}{ Number of nodes } & & & & & \multirow[b]{2}{*}{ Mean } & \multirow[b]{2}{*}{ Std } \\
\hline & & & & & Mean & Std & Mean & Std & Mean & Std & \multicolumn{2}{|c|}{ Mean Std } & & \\
\hline & \multirow{33}{*}{0.60} & \multirow{15}{*}{0.40} & \multirow[t]{3}{*}{1.010} & -1.100 & 141.16 & 266.80 & 4292168 & 7969042 & 1.210 & 2.149 & 0.640 & 1.138 & 0.288 & 0.739 \\
\hline & & & & -1.010 & 126.62 & 238.58 & 3845431 & 6989210 & 1.435 & 3.493 & 1.171 & 3.340 & 0.079 & 0.141 \\
\hline & & & & -1.001 & 126.70 & 238.03 & 3845492 & 6967975 & 0.523 & 0.745 & 0.959 & 2.018 & 0.044 & 0.112 \\
\hline & & & \multirow[t]{3}{*}{1.001} & -1.100 & 139.34 & 263.09 & 4235574 & 7853433 & 0.859 & 1.420 & 0.629 & 1.110 & 0.191 & 0.568 \\
\hline & & & & -1.010 & 125.43 & 235.35 & 3810783 & 6895185 & 0.870 & 1.285 & 0.385 & 0.569 & 0.045 & 0.084 \\
\hline & & & & -1.001 & 125.08 & 234.49 & 3795435 & 6861682 & 0.553 & 1.263 & 0.563 & 1.247 & 0.110 & 0.167 \\
\hline & & & \multirow[t]{3}{*}{1.100} & -1.100 & 28.56 & 30.78 & 904301 & 959846 & 0.332 & 0.719 & 0.777 & 1.560 & 0.647 & 1.437 \\
\hline & & & & -1.010 & 28.18 & 34.87 & 899501 & 1104145 & 0.285 & 0.463 & 0.220 & 0.386 & 0.269 & 0.536 \\
\hline & & & & -1.001 & 29.79 & 34.57 & 956497 & 1095900 & 0.650 & 1.593 & 0.394 & 0.805 & 0.090 & 0.145 \\
\hline & & & \multirow[t]{3}{*}{1.010} & -1.100 & 25.62 & 27.50 & 815854 & 864274 & 0.741 & 1.199 & 0.594 & 1.163 & 0.450 & 1.150 \\
\hline & & & & -1.010 & 25.00 & 30.48 & 802771 & 973960 & 1.041 & 2.791 & 0.214 & 0.289 & 0.141 & 0.189 \\
\hline & & & & -1.001 & 26.43 & 30.25 & 853055 & 968158 & 0.424 & 0.648 & 0.200 & 0.265 & 0.108 & 0.139 \\
\hline & & & \multirow[t]{3}{*}{1.001} & -1.100 & 25.29 & 27.15 & 805707 & 853584 & 0.304 & 0.445 & 0.502 & 0.886 & 0.122 & 0.178 \\
\hline & & & & -1.010 & 24.70 & 30.06 & 793335 & 961321 & 0.881 & 2.390 & 0.813 & 2.275 & 0.105 & 0.160 \\
\hline & & & & -1.001 & 26.15 & 29.84 & 844493 & 955428 & 0.845 & 1.978 & 0.576 & 0.928 & 0.186 & 0.203 \\
\hline & & 0.50 & 1.100 & -1.100 & 37.75 & 48.89 & 1206598 & 1540373 & 0.205 & 0.261 & 0.181 & 0.302 & 0.095 & 0.199 \\
\hline & & & & -1.010 & 39.24 & 51.55 & 1246699 & 1622670 & 0.426 & 0.637 & 0.289 & 0.306 & 0.167 & 0.299 \\
\hline & & & & -1.001 & 39.60 & 51.93 & 1257479 & 1634715 & 0.318 & 0.558 & 0.496 & 0.822 & 0.117 & 0.176 \\
\hline & & & 1.010 & -1.100 & 33.68 & 43.19 & 1080679 & 1369768 & 0.547 & 1.110 & 0.109 & 0.141 & 0.090 & 0.101 \\
\hline & & & & -1.010 & 34.55 & 44.66 & 1101296 & 1415690 & 0.240 & 0.413 & 0.762 & 1.152 & 0.168 & 0.399 \\
\hline & & & & -1.001 & 34.69 & 44.80 & 1105004 & 1420683 & 0.532 & 0.737 & 0.280 & 0.323 & 0.239 & 0.479 \\
\hline & & & 1.001 & -1.100 & 33.38 & 42.89 & 1071473 & 1360381 & 0.334 & 0.669 & 0.810 & 1.721 & 0.118 & 0.296 \\
\hline & & & & -1.010 & 34.08 & 43.99 & 1086439 & 1395117 & 0.585 & 0.916 & 0.383 & 0.587 & 0.183 & 0.424 \\
\hline & & & & -1.001 & 34.24 & 44.12 & 1091361 & 1400239 & 0.320 & 0.531 & 0.550 & 1.006 & 0.177 & 0.413 \\
\hline & & 0.60 & 1.100 & -1.100 & 51.00 & 72.66 & 1619576 & 2279361 & 0.427 & 0.609 & 0.301 & 0.360 & 0.234 & 0.456 \\
\hline & & & & -1.010 & 48.94 & 73.35 & 1532443 & 2267957 & 0.687 & 0.778 & 0.367 & 0.596 & 0.157 & 0.362 \\
\hline & & & & -1.001 & 48.77 & 73.14 & 1524128 & 2255341 & 0.530 & 0.750 & 0.351 & 0.477 & 0.238 & 0.387 \\
\hline & & & 1.010 & -1.100 & 45.80 & 64.27 & 1454993 & 2015529 & 0.342 & 0.579 & 0.472 & 0.701 & 0.247 & 0.650 \\
\hline & & & & -1.010 & 43.98 & 64.28 & 1379773 & 1998789 & 0.467 & 0.735 & 0.473 & 0.653 & 0.203 & 0.326 \\
\hline & & & & -1.001 & 43.52 & 63.35 & 1361289 & 1959494 & 0.542 & 0.742 & 0.332 & 0.513 & 0.410 & 0.634 \\
\hline & & & 1.001 & -1.100 & 45.40 & 63.51 & 1442718 & 1991271 & 0.344 & 0.400 & 0.650 & 0.924 & 0.146 & 0.206 \\
\hline & & & & -1.010 & 43.38 & 63.29 & 1360805 & 1967468 & 0.479 & 0.758 & 0.611 & 0.744 & 0.146 & 0.244 \\
\hline & & & & -1.001 & 42.97 & 62.32 & 1344046 & 1927063 & 0.361 & 0.658 & 0.730 & 1.147 & 0.294 & 0.578 \\
\hline & Avera & & & & 190.83 & 332.10 & 5785994 & 9867958 & 0.655 & 1.104 & 0.615 & 1.029 & 0.232 & 0.429 \\
\hline
\end{tabular}

We coded all the algorithms in Fortran using the Compaq Visual Fortran version 6.6 and performed the experiments on a personal computer powered by an Intel Core2 Quad CPU $2.66 \mathrm{GHz}$ with $4 \mathrm{~GB}$ of RAM and operating under Windows $\mathrm{XP}$. The job processing times was generated from a uniform distribution over the integers 1-100. The weights of the jobs from the first agent were generated from another uniform distribution over the integers 1-100. In addition, the due date $d_{k}$ of $J_{k}$ of $A G_{1}$ was generated from a uniform distribution over the integers between $T(1-\tau-R / 2)$ and $T(1-\tau+R / 2)$, where $T$ is the total normal processing time of the $n$ jobs, that is, $T=\sum_{i=1}^{n} p_{i}$ as proposed by Fisher [33]. $\tau$ took the values $0.4,0.5$, and 0.6 , while $R$ took the values $0.4,0.5$, and 0.6 . We fixed the proportions of the jobs of agent $A G_{1}$ at pro $=0.4$ and 0.6 in the tests.

For the branch-and-bound algorithm, we recorded the average and standard deviation of the number of nodes as well as the average and standard deviation of the execution time (in seconds). For the SA heuristics, we recorded the mean and standard deviation percentage errors. We calculate the percentage error of a solution produced by a heuristic algorithm as follows:

$$
\frac{V-V^{*}}{V^{*}}
$$

where $V$ and $V^{*}$ are the total weighted completion time of the heuristic and the optimal solutions, respectively. We did not record the computational time of the heuristic algorithms because for all of the algorithms it was less than a second CPU time in generating solutions.

We conducted the computational experiments in two parts. In the first part of the experiments, we fixed the number of jobs at 10 and 15. We set the learning index at 1.001, 1.01, and 1.1, and the deteriorating index at $-1.1,-1.01$, and -1.001 . As a result, we examined 324 experimental situations. We randomly generated a set of 20 instances for each situation. 
TABLE 5: RPD of SA algorithms $(n=25)$.

\begin{tabular}{|c|c|c|c|c|c|c|c|c|c|c|c|c|c|c|}
\hline \multirow{3}{*}{ Pro $R$} & \multirow{3}{*}{$a$} & \multirow{3}{*}{$\beta$} & \multicolumn{4}{|c|}{$\tau=0.4$} & \multicolumn{4}{|c|}{$\tau=0.5$} & \multicolumn{4}{|c|}{$\tau=0.6$} \\
\hline & & & $\mathrm{SA}_{1}$ & $\mathrm{SA}_{2}$ & $\mathrm{~S} A$ & & $\mathrm{SA}_{1}$ & $\mathrm{SA}_{2}$ & SA & & $\mathrm{SA}_{1}$ & $\mathrm{~S}$ & & $\mathrm{SA}_{3}$ \\
\hline & & & Mean Std & Mean Std & Mean & Std & Mean Std & Mean Std & Mean & Std & Mean Std & Mean & Std & Mean Std \\
\hline
\end{tabular}

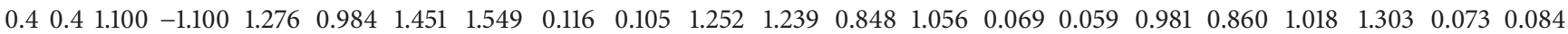
$\begin{array}{lllllllllllllllllll}-1.010 & 2.237 & 3.030 & 1.220 & 1.135 & 0.202 & 0.222 & 1.171 & 1.545 & 1.062 & 1.081 & 0.147 & 0.221 & 1.319 & 1.701 & 1.235 & 1.045 & 0.063 & 0.089\end{array}$ $\begin{array}{lllllllllllllllllllll}-1.001 & 1.327 & 1.370 & 1.320 & 1.232 & 0.137 & 0.177 & 1.442 & 1.389 & 1.295 & 1.792 & 0.156 & 0.236 & 1.381 & 1.328 & 1.529 & 2.077 & 0.050 & 0.065\end{array}$

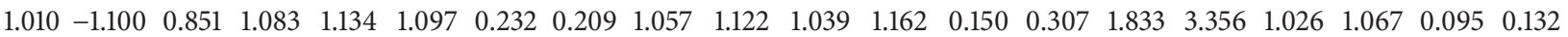
$\begin{array}{llllllllllllllllllllll}-1.010 & 1.442 & 1.442 & 1.356 & 1.360 & 0.163 & 0.162 & 1.280 & 1.100 & 1.707 & 1.473 & 0.060 & 0.090 & 1.266 & 1.500 & 0.905 & 0.905 & 0.096 & 0.166\end{array}$ $\begin{array}{lllllllllllllllllll}-1.001 & 1.251 & 1.288 & 1.343 & 1.579 & 0.166 & 0.136 & 1.246 & 1.219 & 1.014 & 1.238 & 0.111 & 0.135 & 1.119 & 0.939 & 1.040 & 1.151 & 0.046 & 0.056\end{array}$

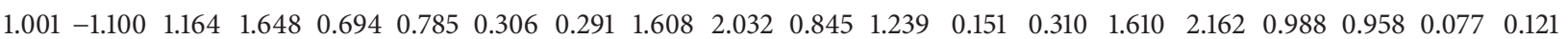
$\begin{array}{llllllllllllllllllll}-1.010 & 1.226 & 1.265 & 1.938 & 1.310 & 0.208 & 0.261 & 1.547 & 1.447 & 1.443 & 1.432 & 0.121 & 0.200 & 0.950 & 1.099 & 0.988 & 1.014 & 0.088 & 0.128\end{array}$ $\begin{array}{lllllllllllllllllll}-1.001 & 1.127 & 1.132 & 1.309 & 1.533 & 0.245 & 0.316 & 0.978 & 1.244 & 1.078 & 0.837 & 0.120 & 0.161 & 0.921 & 0.828 & 1.282 & 1.554 & 0.103 & 0.154\end{array}$ $\begin{array}{lllllllllllllllllllll}0.5 & 1.100 & -1.100 & 1.279 & 1.509 & 1.655 & 1.523 & 0.193 & 0.305 & 1.023 & 0.814 & 1.100 & 0.819 & 0.076 & 0.081 & 1.069 & 0.696 & 0.666 & 0.421 & 0.091 & 0.153\end{array}$ $\begin{array}{lllllllllllllllllllll}-1.010 & 1.406 & 2.069 & 1.301 & 1.285 & 0.206 & 0.317 & 1.476 & 1.692 & 1.976 & 1.672 & 0.096 & 0.159 & 0.785 & 1.046 & 0.910 & 1.003 & 0.086 & 0.130\end{array}$ $\begin{array}{llllllllllllllllllllll}-1.001 & 1.688 & 2.166 & 1.767 & 1.884 & 0.256 & 0.296 & 1.383 & 1.223 & 1.436 & 1.185 & 0.127 & 0.195 & 1.407 & 1.444 & 1.236 & 1.421 & 0.056 & 0.059\end{array}$

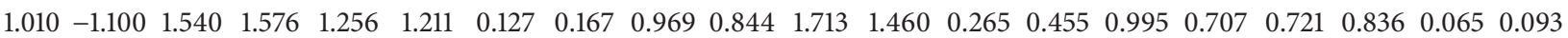
$\begin{array}{lllllllllllllllllll}-1.010 & 2.135 & 2.079 & 2.476 & 2.687 & 0.127 & 0.166 & 1.398 & 0.922 & 1.069 & 1.186 & 0.110 & 0.240 & 1.320 & 1.600 & 0.984 & 1.009 & 0.111 & 0.161\end{array}$ $\begin{array}{lllllllllllllllllll}-1.001 & 1.572 & 2.298 & 1.697 & 1.159 & 0.184 & 0.223 & 1.476 & 1.193 & 1.381 & 1.002 & 0.100 & 0.204 & 1.227 & 2.268 & 0.696 & 1.131 & 0.137 & 0.224\end{array}$

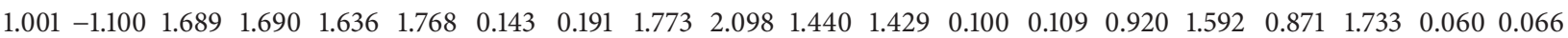
$\begin{array}{llllllllllllllllllll}-1.010 & 1.811 & 1.276 & 1.275 & 1.484 & 0.167 & 0.195 & 1.515 & 1.000 & 1.207 & 1.194 & 0.140 & 0.303 & 1.204 & 1.728 & 1.271 & 1.913 & 0.070 & 0.111\end{array}$ $\begin{array}{llllllllllllllllllll}-1.001 & 1.977 & 2.812 & 1.330 & 1.354 & 0.134 & 0.177 & 1.547 & 1.560 & 1.746 & 1.463 & 0.167 & 0.335 & 1.476 & 1.614 & 1.305 & 1.717 & 0.094 & 0.142\end{array}$ $\begin{array}{lllllllllllllllllllll}0.6 & 1.100 & -1.100 & 1.364 & 1.807 & 1.177 & 0.992 & 0.231 & 0.379 & 1.603 & 1.655 & 1.387 & 1.473 & 0.111 & 0.225 & 1.433 & 1.494 & 1.084 & 1.241 & 0.142 & 0.196\end{array}$ $\begin{array}{lllllllllllllllllll}-1.010 & 2.173 & 3.049 & 2.219 & 2.145 & 0.176 & 0.265 & 1.338 & 1.514 & 2.078 & 2.272 & 0.100 & 0.150 & 1.182 & 1.204 & 1.034 & 1.010 & 0.132 & 0.273\end{array}$ $\begin{array}{lllllllllllllllllllll}-1.001 & 2.136 & 2.370 & 1.742 & 1.657 & 0.127 & 0.170 & 1.853 & 1.322 & 1.712 & 1.909 & 0.078 & 0.098 & 0.839 & 0.793 & 0.962 & 1.013 & 0.081 & 0.100\end{array}$

$\begin{array}{lllllllllllllllllllll}1.010 & -1.100 & 1.898 & 1.644 & 1.936 & 2.001 & 0.171 & 0.230 & 1.481 & 1.649 & 1.543 & 1.681 & 0.185 & 0.342 & 0.755 & 0.548 & 0.855 & 1.082 & 0.104 & 0.158\end{array}$ $\begin{array}{llllllllllllllllllll}-1.010 & 2.004 & 2.399 & 0.903 & 0.734 & 0.121 & 0.125 & 2.320 & 2.198 & 1.635 & 1.670 & 0.179 & 0.342 & 0.901 & 1.147 & 0.952 & 1.035 & 0.181 & 0.429\end{array}$ $\begin{array}{lllllllllllllllllll}-1.001 & 1.678 & 1.944 & 1.885 & 2.021 & 0.080 & 0.068 & 1.602 & 1.614 & 2.044 & 2.312 & 0.052 & 0.039 & 0.916 & 0.856 & 0.735 & 0.738 & 0.084 & 0.114\end{array}$

$\begin{array}{llllllllllllllllllll}1.001 & -1.100 & 1.144 & 0.949 & 1.375 & 1.479 & 0.182 & 0.468 & 1.678 & 1.463 & 1.971 & 1.689 & 0.062 & 0.057 & 0.720 & 0.605 & 0.868 & 0.850 & 0.137 & 0.217\end{array}$ $\begin{array}{lllllllllllllllllll}-1.010 & 3.540 & 3.913 & 2.176 & 1.990 & 0.080 & 0.080 & 2.338 & 2.018 & 1.620 & 1.828 & 0.176 & 0.372 & 1.035 & 1.056 & 0.913 & 0.985 & 0.117 & 0.238\end{array}$ $\begin{array}{lllllllllllllllllllll}-1.001 & 2.201 & 1.911 & 2.537 & 3.343 & 0.109 & 0.130 & 2.001 & 1.998 & 1.986 & 1.543 & 0.070 & 0.081 & 1.116 & 1.083 & 0.897 & 1.308 & 0.084 & 0.123\end{array}$ $\begin{array}{llllllllllllllllllllll}0.6 & 0.4 & 1.100 & -1.100 & 1.350 & 1.721 & 1.661 & 1.605 & 0.186 & 0.142 & 0.873 & 0.883 & 0.806 & 0.757 & 0.302 & 0.600 & 0.572 & 0.338 & 0.484 & 0.259 & 0.120 & 0.142\end{array}$ $\begin{array}{lllllllllllllllllll}-1.010 & 2.082 & 2.202 & 1.762 & 1.940 & 0.208 & 0.231 & 1.461 & 1.621 & 1.312 & 1.793 & 0.156 & 0.172 & 0.779 & 1.217 & 0.786 & 0.831 & 0.134 & 0.220\end{array}$ $\begin{array}{llllllllllllllllllll}-1.001 & 1.085 & 1.635 & 1.410 & 2.158 & 0.207 & 0.269 & 1.587 & 1.412 & 0.681 & 0.567 & 0.188 & 0.245 & 0.804 & 0.614 & 0.693 & 0.670 & 0.148 & 0.223\end{array}$

$\begin{array}{lllllllllllllllllllll}1.010 & -1.100 & 0.814 & 0.901 & 1.219 & 1.720 & 0.192 & 0.234 & 0.884 & 0.826 & 0.791 & 0.749 & 0.202 & 0.337 & 0.717 & 0.491 & 0.519 & 0.449 & 0.097 & 0.091\end{array}$

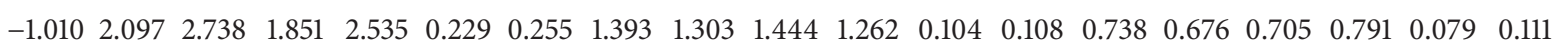
$\begin{array}{lllllllllllllllllllll}-1.001 & 1.563 & 1.479 & 1.750 & 2.206 & 0.163 & 0.252 & 1.402 & 1.544 & 1.064 & 0.906 & 0.153 & 0.220 & 0.891 & 1.148 & 0.638 & 0.928 & 0.113 & 0.156\end{array}$

$\begin{array}{llllllllllllllllllll}1.001 & -1.100 & 1.734 & 1.894 & 1.375 & 1.568 & 0.224 & 0.255 & 0.807 & 0.709 & 1.012 & 1.378 & 0.196 & 0.303 & 1.025 & 1.557 & 0.479 & 0.309 & 0.106 & 0.120\end{array}$ $\begin{array}{llllllllllllllllllll}-1.010 & 1.943 & 1.995 & 1.199 & 1.521 & 0.231 & 0.393 & 1.234 & 1.251 & 1.574 & 2.098 & 0.094 & 0.106 & 0.681 & 0.505 & 1.006 & 1.360 & 0.123 & 0.141\end{array}$ $\begin{array}{llllllllllllllllllllll}-1.001 & 1.322 & 1.699 & 1.694 & 2.141 & 0.210 & 0.232 & 0.980 & 0.753 & 0.931 & 0.725 & 0.120 & 0.139 & 0.629 & 0.766 & 1.072 & 1.252 & 0.067 & 0.090\end{array}$ $\begin{array}{lllllllllllllllllllll}0.5 & 1.100 & -1.100 & 1.744 & 2.154 & 1.746 & 1.783 & 0.193 & 0.239 & 1.001 & 0.912 & 0.905 & 0.710 & 0.055 & 0.048 & 0.602 & 0.615 & 0.859 & 0.643 & 0.185 & 0.225\end{array}$ $\begin{array}{lllllllllllllllllll}-1.010 & 1.667 & 1.727 & 0.977 & 0.852 & 0.218 & 0.292 & 0.911 & 0.975 & 1.187 & 1.459 & 0.275 & 0.315 & 0.975 & 0.742 & 0.658 & 0.535 & 0.123 & 0.128\end{array}$ $\begin{array}{llllllllllllllllllllll}-1.001 & 1.563 & 2.255 & 1.449 & 1.930 & 0.252 & 0.414 & 1.310 & 1.307 & 1.282 & 1.488 & 0.156 & 0.209 & 0.667 & 0.489 & 0.650 & 0.480 & 0.178 & 0.176\end{array}$

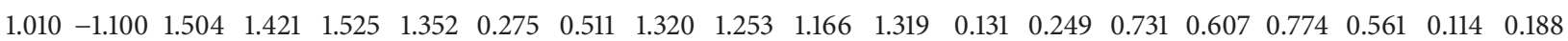
$\begin{array}{llllllllllllllllllll}-1.010 & 1.958 & 3.191 & 1.043 & 0.955 & 0.111 & 0.178 & 1.224 & 1.692 & 0.985 & 0.759 & 0.121 & 0.248 & 0.687 & 0.760 & 0.728 & 0.625 & 0.146 & 0.213\end{array}$ $\begin{array}{llllllllllllllllllll}-1.001 & 1.702 & 2.288 & 1.771 & 1.651 & 0.139 & 0.164 & 1.584 & 1.506 & 1.310 & 1.067 & 0.100 & 0.146 & 0.806 & 0.844 & 0.857 & 0.803 & 0.140 & 0.235\end{array}$

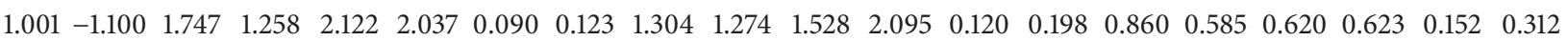
$\begin{array}{lllllllllllllllllll}-1.010 & 1.817 & 2.022 & 2.203 & 2.099 & 0.105 & 0.192 & 0.944 & 0.910 & 1.855 & 1.977 & 0.155 & 0.247 & 1.008 & 1.120 & 0.856 & 0.787 & 0.160 & 0.195\end{array}$ $\begin{array}{lllllllllllllllllll}-1.001 & 1.783 & 1.698 & 1.428 & 1.175 & 0.149 & 0.150 & 1.500 & 1.726 & 1.126 & 1.490 & 0.111 & 0.130 & 1.002 & 1.021 & 1.005 & 0.718 & 0.091 & 0.126\end{array}$ 
TABLE 5: Continued.

\begin{tabular}{|c|c|c|c|c|c|c|c|c|c|c|c|c|c|c|c|c|c|c|c|c|}
\hline \multirow{3}{*}{ Pro } & \multirow{3}{*}{$a$} & \multirow{3}{*}{$\beta$} & \multicolumn{6}{|c|}{$\tau=0.4$} & \multicolumn{6}{|c|}{$\tau=0.5$} & \multicolumn{6}{|c|}{$\tau=0.6$} \\
\hline & & & \multicolumn{2}{|c|}{$\mathrm{SA}_{1}$} & \multicolumn{2}{|c|}{$\mathrm{SA}_{2}$} & \multicolumn{2}{|c|}{$\mathrm{SA}_{3}$} & \multicolumn{2}{|c|}{$\mathrm{SA}_{1}$} & \multicolumn{2}{|c|}{$\mathrm{SA}_{2}$} & \multicolumn{2}{|c|}{$\mathrm{SA}_{3}$} & \multicolumn{2}{|c|}{$\mathrm{SA}_{1}$} & \multicolumn{2}{|c|}{$\mathrm{SA}_{2}$} & \multicolumn{2}{|c|}{$\mathrm{SA}_{3}$} \\
\hline & & & Tean & Std & Mean & Std & Mean & Std & Mean & Std & Mean & Std & Mean & Std & Mean & Std & Mean & Std & Mean & Std \\
\hline \multirow[t]{9}{*}{0.6} & 1.100 & -1.100 & 2.001 & 2.296 & 2.037 & 1.611 & 0.173 & 0.259 & 1.719 & 2.278 & 1.438 & 1.670 & 0.200 & 0.191 & 0.797 & 0.696 & 0.928 & 0.704 & 0.134 & 0.224 \\
\hline & & -1.010 & 933 & 1.773 & 1.871 & 2.590 & 0.179 & 0.277 & 0.608 & 0.490 & 1.129 & 1.337 & 0.238 & 0.269 & 1.196 & 1.227 & 1.045 & 1.245 & 0.144 & 0.180 \\
\hline & & -1.001 & 2.745 & 2.391 & 1.614 & 2.092 & 0.149 & 0.400 & 1.527 & 1.599 & & 1.689 & 0.215 & & 1.063 & 1.289 & 1.134 & 1.092 & & 0.319 \\
\hline & 1.010 & -1.100 & 176 & 2.464 & 2.824 & 2.469 & 0.131 & 0.191 & 1.229 & 1.199 & 0.999 & 0.781 & 0.151 & 0.237 & 0.739 & 0.692 & 0.780 & 0.578 & 0.136 & 0.187 \\
\hline & & -1.010 & 1.813 & 2.478 & 2.536 & 2.103 & 0.158 & 0.218 & 1.864 & 1.945 & 0.913 & 0.774 & 0.156 & 0.205 & 1.438 & 2.337 & 0.958 & 1.094 & 0.099 & 0.140 \\
\hline & & -1.001 & 2.404 & 2.671 & 1.896 & 2.346 & 0.129 & 0.194 & 1.136 & 1.136 & 1.946 & 1.998 & 0.187 & 0.377 & 1.045 & 0.954 & 1.299 & 1.311 & 0.116 & 0.172 \\
\hline & 1.001 & -1.100 & 1.952 & 2.320 & 1.588 & 1.909 & 0.144 & 0.165 & 1.148 & 0.953 & 1.114 & 1.362 & 0.232 & 0.589 & 1.016 & 0.955 & 0.758 & 0.606 & 0.154 & 0.227 \\
\hline & & -1.010 & 2.130 & 3.139 & 2.191 & 2.195 & 0.120 & 0.165 & 1.518 & 2.083 & 1.488 & 1.477 & 0.184 & 0.245 & 1.402 & 1.157 & 1.490 & 1.744 & 0.205 & 0.374 \\
\hline & & -1.001 & 1.813 & 2.394 & 2.407 & 3.146 & 0.100 & 0.156 & 1.722 & 2.895 & 1.792 & 1.745 & 0.316 & 0.4 & 1.342 & 2.332 & 1.205 & 0.912 & 0.153 & 0.276 \\
\hline & & & 1.733 & 1.980 & 1.653 & 1.740 & 0.171 & 0.229 & 1.380 & 1.399 & 1.340 & 1.380 & 0.146 & 0.232 & 1.017 & 1.129 & 0.925 & 0.989 & 0.113 & 0.170 \\
\hline
\end{tabular}

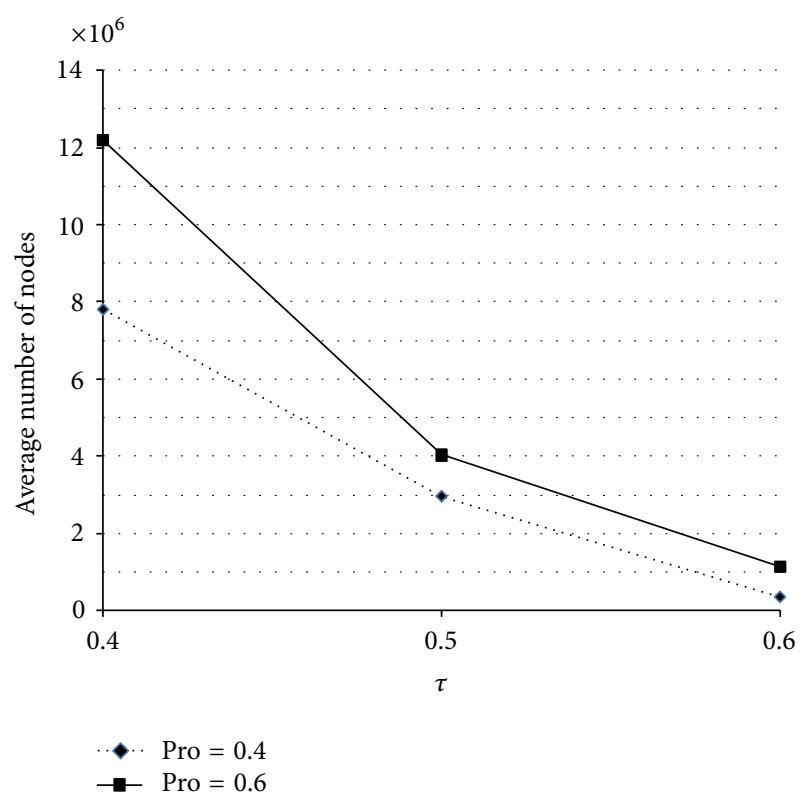

FIgURE 1: Performance of the branch-and-bound algorithm $(n=15)$.

Tables 1, 2, 3, and 4 report the results, which include the CPU time (mean and standard deviation) and the number of nodes for the branch-and-bound algorithm.

As for the performance of the branch-and-bound algorithm, we see from Figure 1 and Table 1 that the number of nodes and the mean CPU time increase when $n$ becomes bigger. When the proportion of the jobs of agent $A G_{1}$ is smaller ( pro $=0.4$ ), the number of nodes is obviously more than that with a bigger one as shown in Figure 2. In addition, the instances with a bigger value of $\tau$ (e.g., $\tau=0.5$ and 0.6 ) are easier to solve than those with a smaller one. Tables 1-4 also show that the mean error percentages of $\mathrm{SA}_{3}$ (between $0.104 \%$ and $0.335 \%$ ) are lower than those of $\mathrm{SA}_{1}$ (between $0.277 \%$ and $0.832 \%$ ) and those of $\mathrm{SA}_{2}$ (between $0.272 \%$ and $0.730 \%$.) Moreover, the standard deviations of the percentage error follow the same pattern. In particular, the standard deviations of the percentage error of $\mathrm{SA}_{1}, \mathrm{SA}_{2}$, and $\mathrm{SA}_{3}$ were

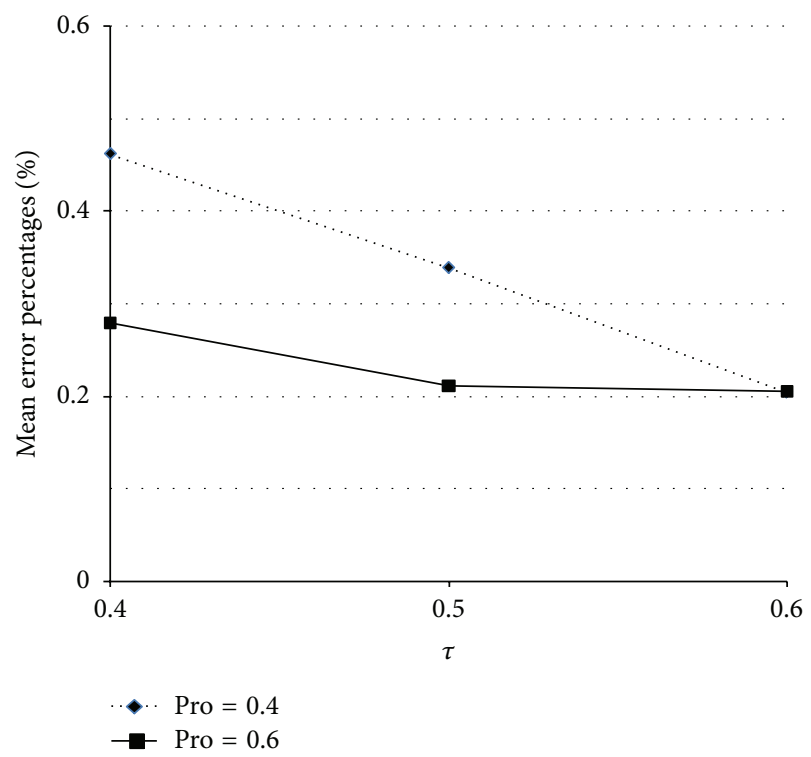

FIgURE 2: Performance of $\mathrm{SA}_{3}$ algorithm $(n=15)$.

between $0.870 \%$ and $1.464 \%, 0.841 \%$ and $1.253 \%$, and $0.352 \%$ and $0.588 \%$, respectively. This indicates that the performance of $\mathrm{SA}_{3}$ is better than that of the other two. In addition, the means error percentages of $\mathrm{SA}_{3}$ are affected by the parameters $\tau$ and pro. In particular, it was up to $0.462 \%$ when the value of $\tau$ became smaller (at $\tau=0.4$ ) or pro was 0.6 (see Figure 2 and Tables 1-4). However, the mean error percentages of $\mathrm{SA}_{3}$ were all below $0.5 \%$. The results also indicate that the impact of the learning or deteriorating effect is insignificant.

In the second part of the experiments, we further assessed the performance of the proposed SA heuristics in solving instances with large numbers of jobs. Given the fact that it is not easy to generate a feasible solution when $n$ becomes lager, we set $n$ at 25 and 30, fixed the parameters at the values as those used for the small job-size design, and set the proportion of the jobs of agent $A G_{1}$ at pro $=0.4$ and 0.6 in the tests. We set the learning index at 1.001, 1.01, and 1.1 , and the deteriorating index at $-1.1,-1.01$, and -1.001 . 
TABLE 6: RPD of SA algorithms $(n=30)$.

\begin{tabular}{|c|c|c|c|c|c|c|c|c|c|c|c|}
\hline \multirow{3}{*}{ Pro $R$} & \multirow{3}{*}{$a$} & \multirow{3}{*}{$\beta$} & \multicolumn{3}{|c|}{$\tau=0.4$} & \multicolumn{3}{|c|}{$\tau=0.5$} & \multicolumn{3}{|c|}{$\tau=0.6$} \\
\hline & & & $\mathrm{SA}_{1}$ & $\mathrm{SA}_{2}$ & $\mathrm{SA}_{3}$ & $\mathrm{SA}_{1}$ & $\mathrm{SA}_{2}$ & $\mathrm{SA}_{3}$ & $\mathrm{SA}_{1}$ & $\mathrm{SA}_{2}$ & $\mathrm{SA}_{3}$ \\
\hline & & & Iean Std & Mean Std & Mean Std & Mean Std & Mean Std & Mean Std & Mean Std & Mean Std & Mean Std \\
\hline
\end{tabular}

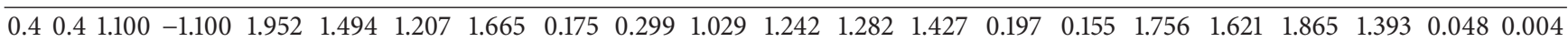
$\begin{array}{lllllllllllllllllll}-1.010 & 1.587 & 1.564 & 1.636 & 1.632 & 0.155 & 0.195 & 1.820 & 2.590 & 0.962 & 0.721 & 0.098 & 0.071 & 0.889 & 1.282 & 0.864 & 1.280 & 0.096 & 0.096\end{array}$

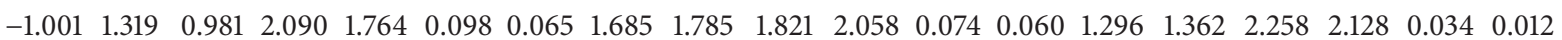

$\begin{array}{lllllllllllllllllllll}1.010 & -1.100 & 2.153 & 2.579 & 1.025 & 1.349 & 0.120 & 0.073 & 1.373 & 1.427 & 1.176 & 1.723 & 0.200 & 0.302 & 1.592 & 0.730 & 1.442 & 0.570 & 0.047 & 0.003\end{array}$ $\begin{array}{llllllllllllllllllll}-1.010 & 1.638 & 1.050 & 1.800 & 1.358 & 0.091 & 0.052 & 1.522 & 1.427 & 1.269 & 1.071 & 0.058 & 0.030 & 0.987 & 1.372 & 1.222 & 1.423 & 0.042 & 0.002\end{array}$ $\begin{array}{llllllllllllllllllll}-1.001 & 1.584 & 1.474 & 2.365 & 2.117 & 0.182 & 0.194 & 1.908 & 1.890 & 2.039 & 1.723 & 0.067 & 0.049 & 1.328 & 1.644 & 1.945 & 2.166 & 0.073 & 0.050\end{array}$

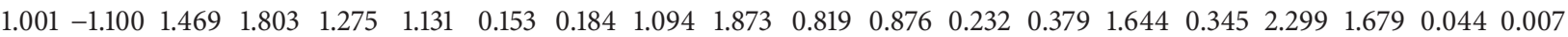
$\begin{array}{llllllllllllllllllll}-1.010 & 2.084 & 1.844 & 1.850 & 1.604 & 0.134 & 0.118 & 1.485 & 1.341 & 2.046 & 2.087 & 0.091 & 0.065 & 1.074 & 0.745 & 1.102 & 0.673 & 0.042 & 0.001\end{array}$ $\begin{array}{lllllllllllllllllll}-1.001 & 1.578 & 1.500 & 1.335 & 1.154 & 0.191 & 0.207 & 1.904 & 1.470 & 2.063 & 1.501 & 0.091 & 0.091 & 1.875 & 2.372 & 1.266 & 1.360 & 0.057 & 0.045\end{array}$

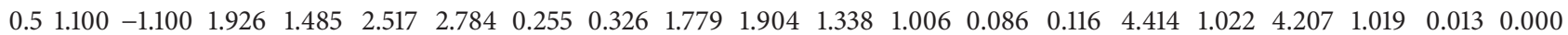
$\begin{array}{lllllllllllllllllll}-1.010 & 2.995 & 2.671 & 1.908 & 1.616 & 0.073 & 0.054 & 3.123 & 3.100 & 1.779 & 1.688 & 0.101 & 0.141 & 2.411 & 1.820 & 1.682 & 1.282 & 0.029 & 0.019\end{array}$ $\begin{array}{lllllllllllllllllll}-1.001 & 2.289 & 1.520 & 2.681 & 2.919 & 0.091 & 0.077 & 2.099 & 1.855 & 1.584 & 1.273 & 0.074 & 0.103 & 1.525 & 1.139 & 1.846 & 1.358 & 0.028 & 0.021\end{array}$

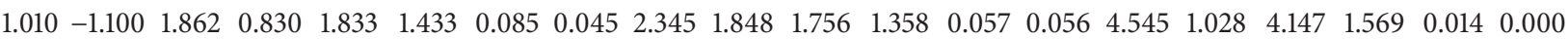
$\begin{array}{lllllllllllllllllll}-1.010 & 2.452 & 2.384 & 2.123 & 1.575 & 0.080 & 0.059 & 1.627 & 1.389 & 2.396 & 1.642 & 0.084 & 0.116 & 1.756 & 1.379 & 1.399 & 1.252 & 0.035 & 0.024\end{array}$ $\begin{array}{lllllllllllllllllll}-1.001 & 2.411 & 3.870 & 2.445 & 1.533 & 0.103 & 0.140 & 2.492 & 2.733 & 2.383 & 2.552 & 0.170 & 0.294 & 1.572 & 1.234 & 1.697 & 1.562 & 0.028 & 0.020\end{array}$

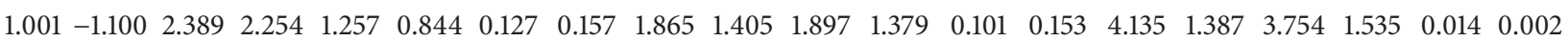
$\begin{array}{llllllllllllllllllll}-1.010 & 2.252 & 2.064 & 2.346 & 1.877 & 0.108 & 0.131 & 2.940 & 3.129 & 2.195 & 1.840 & 0.062 & 0.065 & 1.725 & 1.207 & 1.702 & 1.401 & 0.058 & 0.113\end{array}$ $\begin{array}{lllllllllllllllllll}-1.001 & 2.178 & 2.644 & 2.907 & 2.485 & 0.133 & 0.206 & 1.721 & 1.428 & 1.880 & 1.554 & 0.093 & 0.114 & 1.691 & 1.277 & 1.660 & 1.316 & 0.027 & 0.020\end{array}$

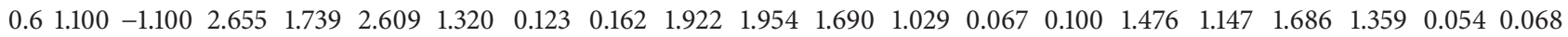
$\begin{array}{llllllllllllllllllll}-1.010 & 2.629 & 3.121 & 1.962 & 1.714 & 0.227 & 0.359 & 2.513 & 1.823 & 2.135 & 1.607 & 0.059 & 0.063 & 1.664 & 1.617 & 1.468 & 0.993 & 0.175 & 0.340\end{array}$ $\begin{array}{lllllllllllllllllll}-1.001 & 2.795 & 2.703 & 2.914 & 2.464 & 0.184 & 0.333 & 2.250 & 1.989 & 1.686 & 1.369 & 0.074 & 0.084 & 1.785 & 1.341 & 1.526 & 1.373 & 0.096 & 0.220\end{array}$

$\begin{array}{llllllllllllllllllll}1.010 & -1.100 & 2.666 & 2.523 & 2.282 & 2.228 & 0.299 & 0.672 & 1.998 & 1.461 & 2.376 & 2.048 & 0.041 & 0.029 & 1.783 & 1.217 & 1.481 & 0.897 & 0.028 & 0.013\end{array}$ $\begin{array}{llllllllllllllllllll}-1.010 & 2.547 & 2.320 & 2.031 & 1.620 & 0.140 & 0.258 & 1.691 & 1.245 & 2.389 & 1.564 & 0.154 & 0.292 & 1.433 & 1.595 & 1.413 & 1.449 & 0.045 & 0.103\end{array}$ $\begin{array}{lllllllllllllllllll}-1.001 & 3.222 & 4.776 & 2.353 & 1.606 & 0.109 & 0.157 & 2.284 & 1.822 & 2.229 & 2.088 & 0.153 & 0.319 & 1.579 & 1.479 & 1.605 & 1.344 & 0.056 & 0.095\end{array}$

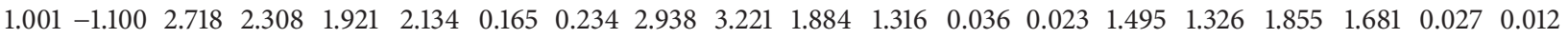
$\begin{array}{lllllllllllllllllll}-1.010 & 2.765 & 2.250 & 2.040 & 1.549 & 0.167 & 0.266 & 2.531 & 1.881 & 1.988 & 1.331 & 0.105 & 0.171 & 1.480 & 1.855 & 1.439 & 1.261 & 0.101 & 0.221\end{array}$ $\begin{array}{lllllllllllllllllllll}-1.001 & 2.293 & 1.809 & 2.085 & 2.169 & 0.106 & 0.141 & 2.008 & 1.436 & 2.117 & 1.815 & 0.092 & 0.204 & 1.634 & 1.306 & 2.103 & 2.015 & 0.027 & 0.035\end{array}$

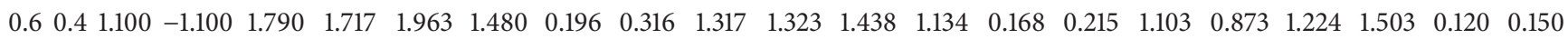
$\begin{array}{lllllllllllllllllll}-1.010 & 1.576 & 1.189 & 1.932 & 1.973 & 0.153 & 0.254 & 1.209 & 1.033 & 1.663 & 1.971 & 0.165 & 0.229 & 1.082 & 1.051 & 1.298 & 1.515 & 0.097 & 0.121\end{array}$ $\begin{array}{lllllllllllllllllllll}-1.001 & 1.499 & 1.558 & 1.503 & 1.429 & 0.138 & 0.196 & 1.023 & 1.077 & 1.430 & 1.186 & 0.148 & 0.213 & 1.034 & 1.219 & 1.162 & 1.237 & 0.132 & 0.199\end{array}$

$\begin{array}{llllllllllllllllllllll}1.010 & -1.100 & 1.062 & 1.047 & 1.711 & 1.688 & 0.132 & 0.104 & 1.807 & 1.929 & 1.382 & 1.176 & 0.097 & 0.099 & 1.049 & 1.029 & 0.771 & 0.459 & 0.078 & 0.104\end{array}$ $\begin{array}{lllllllllllllllllll}-1.010 & 1.430 & 1.360 & 2.181 & 1.577 & 0.179 & 0.243 & 1.163 & 0.981 & 1.425 & 1.567 & 0.198 & 0.152 & 1.077 & 1.046 & 1.087 & 0.781 & 0.153 & 0.216\end{array}$ $\begin{array}{lllllllllllllllllllll}-1.001 & 1.653 & 1.575 & 1.642 & 1.209 & 0.175 & 0.325 & 2.061 & 1.980 & 1.234 & 0.936 & 0.153 & 0.209 & 0.802 & 0.450 & 1.050 & 1.589 & 0.059 & 0.063\end{array}$

$\begin{array}{llllllllllllllllllllll}1.001 & -1.100 & 1.677 & 1.309 & 1.435 & 1.103 & 0.163 & 0.162 & 1.668 & 1.498 & 1.509 & 1.172 & 0.096 & 0.107 & 1.036 & 0.680 & 1.155 & 1.061 & 0.097 & 0.137\end{array}$ $\begin{array}{lllllllllllllllllll}-1.010 & 2.283 & 3.104 & 1.890 & 1.456 & 0.160 & 0.215 & 1.127 & 1.248 & 1.186 & 1.012 & 0.182 & 0.190 & 1.000 & 0.969 & 0.795 & 0.681 & 0.097 & 0.138\end{array}$ $\begin{array}{lllllllllllllllllll}-1.001 & 1.430 & 1.056 & 1.753 & 1.323 & 0.112 & 0.108 & 1.451 & 1.557 & 1.420 & 1.468 & 0.108 & 0.132 & 0.855 & 0.586 & 1.009 & 1.221 & 0.113 & 0.156\end{array}$

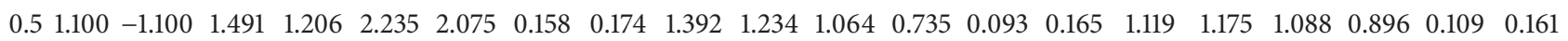
$\begin{array}{lllllllllllllllllll}-1.010 & 1.855 & 1.334 & 1.459 & 1.149 & 0.202 & 0.351 & 1.524 & 1.257 & 1.491 & 1.145 & 0.136 & 0.199 & 0.960 & 0.920 & 1.090 & 0.911 & 0.045 & 0.083\end{array}$ $\begin{array}{llllllllllllllllllll}-1.001 & 2.134 & 2.407 & 1.960 & 1.166 & 0.088 & 0.089 & 2.920 & 2.779 & 2.011 & 2.250 & 0.081 & 0.128 & 1.839 & 2.162 & 1.273 & 1.324 & 0.103 & 0.160\end{array}$

$\begin{array}{llllllllllllllllllll}1.010 & -1.100 & 1.529 & 1.089 & 1.982 & 1.666 & 0.144 & 0.132 & 1.804 & 2.638 & 1.738 & 1.065 & 0.077 & 0.127 & 1.192 & 0.886 & 1.215 & 0.863 & 0.065 & 0.096\end{array}$ $\begin{array}{lllllllllllllllllll}-1.010 & 1.387 & 1.264 & 2.743 & 2.388 & 0.136 & 0.196 & 1.981 & 1.360 & 2.021 & 2.360 & 0.060 & 0.066 & 1.574 & 1.088 & 1.178 & 1.023 & 0.070 & 0.175\end{array}$ $\begin{array}{lllllllllllllllllll}-1.001 & 1.553 & 1.338 & 2.148 & 1.645 & 0.159 & 0.207 & 1.628 & 1.438 & 1.804 & 1.503 & 0.128 & 0.186 & 1.127 & 0.815 & 0.897 & 0.630 & 0.094 & 0.158\end{array}$

$\begin{array}{llllllllllllllllllllll}1.001 & -1.100 & 1.810 & 1.748 & 2.087 & 1.721 & 0.148 & 0.162 & 1.255 & 1.135 & 1.607 & 1.678 & 0.070 & 0.099 & 0.926 & 0.608 & 0.957 & 0.535 & 0.076 & 0.116\end{array}$ $\begin{array}{lllllllllllllllllll}-1.010 & 1.884 & 1.540 & 1.389 & 1.377 & 0.165 & 0.222 & 1.895 & 1.748 & 1.912 & 2.004 & 0.105 & 0.182 & 1.315 & 1.020 & 1.121 & 0.679 & 0.041 & 0.091\end{array}$ $\begin{array}{lllllllllllllllllll}-1.001 & 2.128 & 2.026 & 2.142 & 1.984 & 0.205 & 0.318 & 1.332 & 0.997 & 1.849 & 1.607 & 0.146 & 0.266 & 1.204 & 0.610 & 1.132 & 0.654 & 0.079 & 0.213\end{array}$

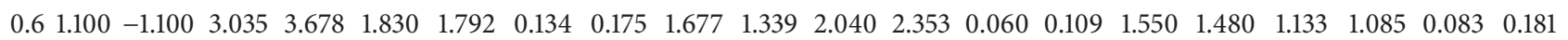
$\begin{array}{lllllllllllllllllll}-1.010 & 1.638 & 1.292 & 1.804 & 1.323 & 0.106 & 0.210 & 1.567 & 1.294 & 1.968 & 1.327 & 0.062 & 0.104 & 1.171 & 0.782 & 0.959 & 0.456 & 0.043 & 0.115\end{array}$

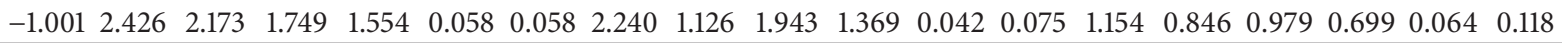


TABLE 6: Continued.

\begin{tabular}{|c|c|c|c|c|c|c|c|c|c|c|c|c|c|c|c|c|c|c|c|c|}
\hline \multirow{3}{*}{ Pro $R$} & & \multirow{3}{*}{$\beta$} & \multicolumn{6}{|c|}{$\tau=0.4$} & \multicolumn{6}{|c|}{$\tau=0.5$} & \multicolumn{6}{|c|}{$\tau=0.6$} \\
\hline & & & \multicolumn{2}{|c|}{$\mathrm{SA}_{1}$} & \multicolumn{2}{|c|}{$\mathrm{SA}_{2}$} & \multicolumn{2}{|c|}{$\mathrm{SA}_{3}$} & \multicolumn{2}{|c|}{$\mathrm{SA}_{1}$} & \multicolumn{2}{|c|}{$\mathrm{SA}_{2}$} & \multicolumn{2}{|c|}{$\mathrm{SA}_{3}$} & \multicolumn{2}{|c|}{$\mathrm{SA}_{1}$} & \multicolumn{2}{|c|}{$\mathrm{SA}_{2}$} & \multicolumn{2}{|c|}{$\mathrm{SA}_{3}$} \\
\hline & & & Mean & Std & Mean & Std & Mean & Std & Mean & Std & Mean & Std & Mean & Std & Mean & Std & Mean & Std & Mean & Std \\
\hline & 1.010 & -1.100 & 2.195 & 2.167 & 1.602 & 1.189 & 0.125 & 0.179 & 1.509 & 0.962 & 1.273 & 1.742 & 0.051 & 0.064 & 1.217 & 0.839 & 1.056 & 0.819 & 0.030 & 0.047 \\
\hline & & -1.010 & 2.499 & 2.162 & 1.141 & 0.841 & 0.095 & 0.148 & 1.860 & 1.068 & 1.845 & 1.465 & 0.031 & 0.046 & 1.072 & 0.641 & 1.563 & 1.337 & 0.030 & 0.095 \\
\hline & & -1.001 & 1.837 & 1.715 & 2.048 & 1.688 & 0.065 & 0.106 & 1.779 & 1.381 & 1.897 & 1.207 & 0.068 & 0.109 & 1.187 & 1.283 & 1.567 & 1.010 & 0.017 & 0.040 \\
\hline & 1.001 & -1.100 & 1.705 & 1.238 & 3.076 & 3.955 & 0.161 & 0.278 & 1.958 & 2.291 & 1.453 & 1.162 & 0.050 & 0.085 & 1.154 & 0.564 & 1.337 & 1.554 & 0.032 & 0.076 \\
\hline & & -1.010 & 935 & 2.334 & 1.617 & 1.652 & 0.040 & 0.029 & 2.130 & 1.566 & 2.181 & 1.594 & 0.049 & 0.104 & 1.766 & 1.831 & 1.803 & 1.169 & 0.017 & 0.028 \\
\hline & & -1.001 & 1.300 & 0.989 & 1.756 & 1.308 & 0.213 & 0.384 & 2.072 & 1.963 & 2.212 & 1.811 & 0.037 & 0.062 & 1.325 & 1.322 & 1.260 & 0.863 & 0.032 & 0.057 \\
\hline \multicolumn{3}{|c|}{ Average } & 2.021 & 1.911 & 1.955 & 1.692 & 0.142 & 0.195 & 1.839 & 1.683 & 1.744 & 1.512 & 0.100 & 0.136 & 1.527 & 1.160 & 1.520 & 1.183 & 0.061 & 0.090 \\
\hline
\end{tabular}

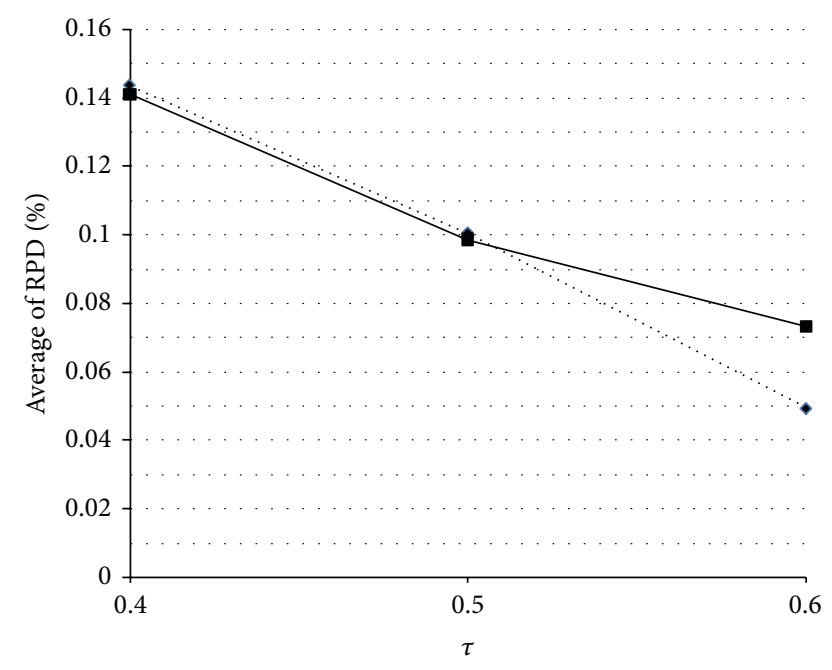

- Pro $=0.4$

FIgURE 3: Performance of $\mathrm{SA}_{3}$ algorithm $(n=30)$.

As a result, we examined 324 experimental situations. We randomly generated a set of 20 instances for each situation. We obtained the relative percentage with respect to the best known solution among $\mathrm{SA}_{1}, \mathrm{SA}_{2}$, and $\mathrm{SA}_{3}$ for each instance. We also recorded the mean execution time and the mean relative percentage deviation for each SA heuristic. We calculate the relative percentage deviation RPD as follows:

$$
\frac{\mathrm{SA}_{i}-\mathrm{SA}^{*}}{\mathrm{SA}^{*}}
$$

where $\mathrm{SA}_{i}$ is the value of the objective function generated by $\mathrm{SA}_{i}$, and $\mathrm{SA}^{*}=\min \left\{\mathrm{SA}_{i}, i=1,2,3\right\}$ is the smallest value of the objective function obtained from the three SA heuristics. Tables 5-6 report the results.

As shown in Tables 5-6, we see that the mean RPDs of $\mathrm{SA}_{1}$ and $\mathrm{SA}_{2}$ become bigger as $n$ increases. In general, the mean RPDs of $\mathrm{SA}_{3}$ are lower than those of $\mathrm{SA}_{1}$ and $\mathrm{SA}_{2}$. Furthermore, Figure 3 and Tables 5-6 show that the RDP mean of $\mathrm{SA}_{3}$ becomes smaller as $\tau$ becomes larger (e.g., $\tau=$ $0.6)$. However, when $\tau=0.6$, the mean RPD of $\mathrm{SA}_{3}$ at pro $=0.4$ was less than that at pro $=0.6$. Figure 4 further shows that when $n=30, \mathrm{SA}_{3}$ has a smaller $\mathrm{RPD}$ value when $\tau$

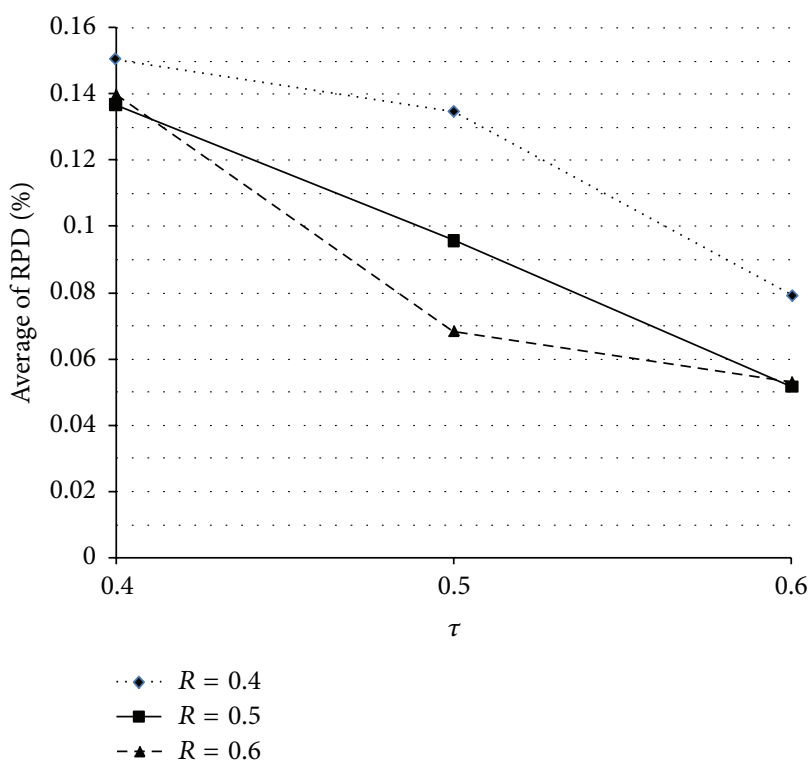

FIGURE 4: Performance of $\mathrm{SA}_{3}$ algorithms $(n=30)$.

or $R$ becomes larger. In addition, all of the mean RPDs of $\mathrm{SA}_{3}$ were less than $0.2 \%$. In sum, $\mathrm{SA}_{3}$ outperforms the other two SA heuristics in terms of both solution accuracy and performance stability.

\section{Conclusions}

In this paper, we study a two-agent single-machine scheduling problem with learning and deteriorating effects simultaneously. The objective is to minimize the total weighted completion time of the jobs of the first agent with the restriction that no tardy job is allowed for the second agent. We develop a branch-and-bound algorithm incorporating several dominant properties and a lower bound to derive the optimal solution. We also propose three simulated annealing algorithms to obtain near-optimal solutions. The computational results show that with the help of the proposed heuristic initial solutions, the branch-and-bound algorithm performs well in terms of the number of nodes and execution time, when the number of jobs is fewer than or equal to 15 . Moreover, the computational experiments also show that the 
proposed $\mathrm{SA}_{3}$ performs well since its mean error percentage was less than $0.4 \%$ for all the tested cases. Further research lies in the devising of efficient and effective methods to solve the problem with significantly larger numbers of jobs.

\section{Acknowledgment}

The author would like to thank the National Science Council of the Republic of China, Taiwan, for financially supporting this paper under the Contract nos. NSC 101-2410-H-264-004 and NSC 102-2410-H-264-003.

\section{References}

[1] S. Browne and U. Yechiali, "Scheduling deteriorating jobs on a single processor," Operations Research, vol. 38, no. 3, pp. 495498, 1990.

[2] D. Biskup, "Single-machine scheduling with learning considerations," European Journal of Operational Research, vol. 115, no. 1, pp. 173-178, 1999.

[3] J. N. D. Gupta and S. K. Gupta, "Single facility scheduling with nonlinear processing times," Computers and Industrial Engineering, vol. 14, no. 4, pp. 387-393, 1988.

[4] B. Alidaee and N. K. Womer, "Scheduling with time dependent processing times: review and extensions," Journal of the Operational Research Society, vol. 50, no. 7, pp. 711-720, 1999.

[5] T. C. E. Cheng, Q. Ding, and B. M. T. Lin, "A concise survey of scheduling with time-dependent processing times," European Journal of Operational Research, vol. 152, no. 1, pp. 1-13, 2004.

[6] T. C. E. Cheng and G. Wang, "Single machine scheduling with learning effect considerations," Annals of Operations Research, vol. 98, no. 1-4, pp. 273-290, 2000.

[7] D. Biskup, "A state-of-the-art review on scheduling with learning effects," European Journal of Operational Research, vol. 188, no. 2, pp. 315-329, 2008.

[8] J.-B. Wang, "A note on scheduling problems with learning effect and deteriorating jobs," International Journal of Systems Science, vol. 37, no. 12, pp. 827-833, 2006.

[9] J.-B. Wang, "Single-machine scheduling problems with the effects of learning and deterioration," Omega, vol. 35, no. 4, pp. 397-402, 2007.

[10] X. Wang and T. C. E. Cheng, "Single-machine scheduling with deteriorating jobs and learning effects to minimize the makespan," European Journal of Operational Research, vol. 178, no. 1, pp. 57-70, 2007.

[11] T. C. E. Cheng, C.-C. Wu, and W.-C. Lee, "Some scheduling problems with deteriorating jobs and learning effects," Computers and Industrial Engineering, vol. 54, no. 4, pp. 972-982, 2008.

[12] T. C. E. Cheng, C. T. Ng, and J. J. Yuan, "Multi-agent scheduling on a single machine with max-form criteria," European Journal of Operational Research, vol. 188, no. 2, pp. 603-609, 2008.

[13] M. D. Toksarı, D. Oron, and E. Güner, "Single machine scheduling problems under the effects of nonlinear deterioration and time-dependent learning," Mathematical and Computer Modelling, vol. 50, no. 3-4, pp. 401-406, 2009.

[14] X. Huang, J.-B. Wang, L.-Y. Wang, W.-J. Gao, and X.-R. Wang, "Single machine scheduling with time-dependent deterioration and exponential learning effect," Computers and Industrial Engineering, vol. 58, no. 1, pp. 58-63, 2010.
[15] S.-R. Cheng, "A single-machine two-agent scheduling problem by GA approach," Asia-Pacific Journal of Operational Research, vol. 29, no. 2, Article ID 1250013, 2012.

[16] D.-C. Li and P.-H. Hsu, "Solving a two-agent single-machine scheduling problem considering learning effect," Computers \& Operations Research, vol. 39, no. 7, pp. 1644-1651, 2012.

[17] A. Agnetis, D. Pacciarelli, and A. Pacifici, "Multi-agent single machine scheduling," Annals of Operations Research, vol. 150, pp. 3-15, 2007.

[18] K. R. Baker and J. C. Smith, "A multiple-criterion model for machine scheduling," Journal of Scheduling, vol. 6, no. 1, pp. 716, 2003.

[19] A. Agnetis, P. B. Mirchandani, D. Pacciarelli, and A. Pacifici, "Scheduling problems with two competing agents," Operations Research, vol. 52, no. 2, pp. 229-242, 2004.

[20] J. J. Yuan, W. P. Shang, and Q. Feng, "A note on the scheduling with two families of jobs," Journal of Scheduling, vol. 8, pp. 537542, 2005.

[21] T. C. E. Cheng, C. T. Ng, and J. J. Yuan, "Multi-agent scheduling on a single machine to minimize total weighted number of tardy jobs," Theoretical Computer Science, vol. 362, no. 1-3, pp. 273281, 2006.

[22] C. T. Ng, T. C. E. Cheng, and J. J. Yuan, "A note on the complexity of the problem of two-agent scheduling on a single machine," Journal of Combinatorial Optimization, vol. 12, no. 4, pp. 387394, 2006.

[23] Y. Yin, S. R. Cheng, T. C. E. Cheng, W. H. Wu, and C. C. $\mathrm{Wu}$, "Two-agent single-machine scheduling with release times and deadlines," International Journal of Shipping and Transport Logistics, 2012.

[24] T. C. E. Cheng, Y.-H. Chung, S.-C. Liao, and W.-C. Lee, “Twoagent singe-machine scheduling with release times to minimize the total weighted completion time," Computers \& Operations Research, vol. 40, no. 1, pp. 353-361, 2013.

[25] P. Liu and L. Tang, "Two-agent scheduling with linear deteriorating jobs on a single machine," in Computing and Combinatorics, vol. 5092 of Lecture Notes in Computer Science, pp. 642650, Springer, Berlin, Germany, 2008.

[26] T. C. E. Cheng, S.-R. Cheng, W.-H. Wu, P.-H. Hsu, and C.$\mathrm{C}$. $\mathrm{Wu}$, "A two-agent single-machine scheduling problem with truncated sum-of-processing-times-based learning considerations," Computers and Industrial Engineering, vol. 60, no. 4, pp. 534-541, 2011.

[27] C. C. Wu, S. K. Huang, and W. C. Lee, "Two-agent scheduling with learning consideration," Computers \& Industrial Engineering, vol. 61, no. 4, pp. 1324-1335, 2011.

[28] S. Kirkpatrick, C. D. Gelatt, Jr., and M. P. Vecchi, "Optimization by simulated annealing," American Association for the Advancement of Science, vol. 220, no. 4598, pp. 671-680, 1983.

[29] R. Stahlbock and S. Voß, "Efficiency considerations for sequencing and scheduling of double-rail-mounted gantry cranes at maritime container terminals," International Journal of Shipping and Transport Logistics, vol. 2, no. 1, pp. 95-123, 2010.

[30] C.-L. Li and K.-W. Pang, "An integrated model for ship routing and berth allocation," International Journal of Shipping and Transport Logistics, vol. 3, no. 3, pp. 245-260, 2011.

[31] M. Nawaz, E. E. Enscore Jr., and I. Ham, "A heuristic algorithm for the m-machine, n-job flow-shop sequencing problem," Omega, vol. 11, no. 1, pp. 91-95, 1983.

[32] D. Ben-Arieh and O. Maimon, "Annealing method for PCB assembly scheduling on two sequential machines," International 
Journal of Computer Integrated Manufacturing, vol. 5, pp. 361367, 1992.

[33] M. L. Fisher, "A dual algorithm for the one-machine scheduling problem," Mathematical Programming, vol. 11, no. 3, pp. 229251, 1976/77. 


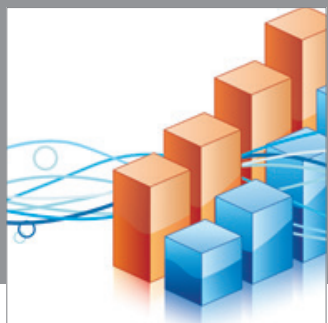

Advances in

Operations Research

mansans

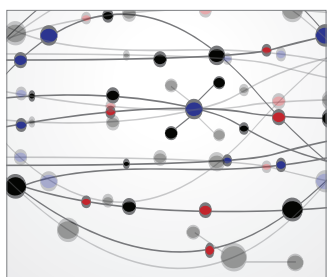

The Scientific World Journal
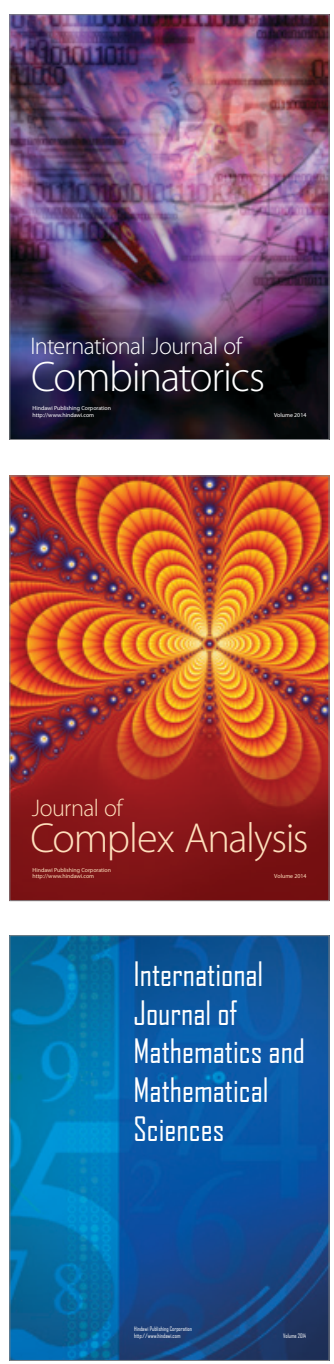
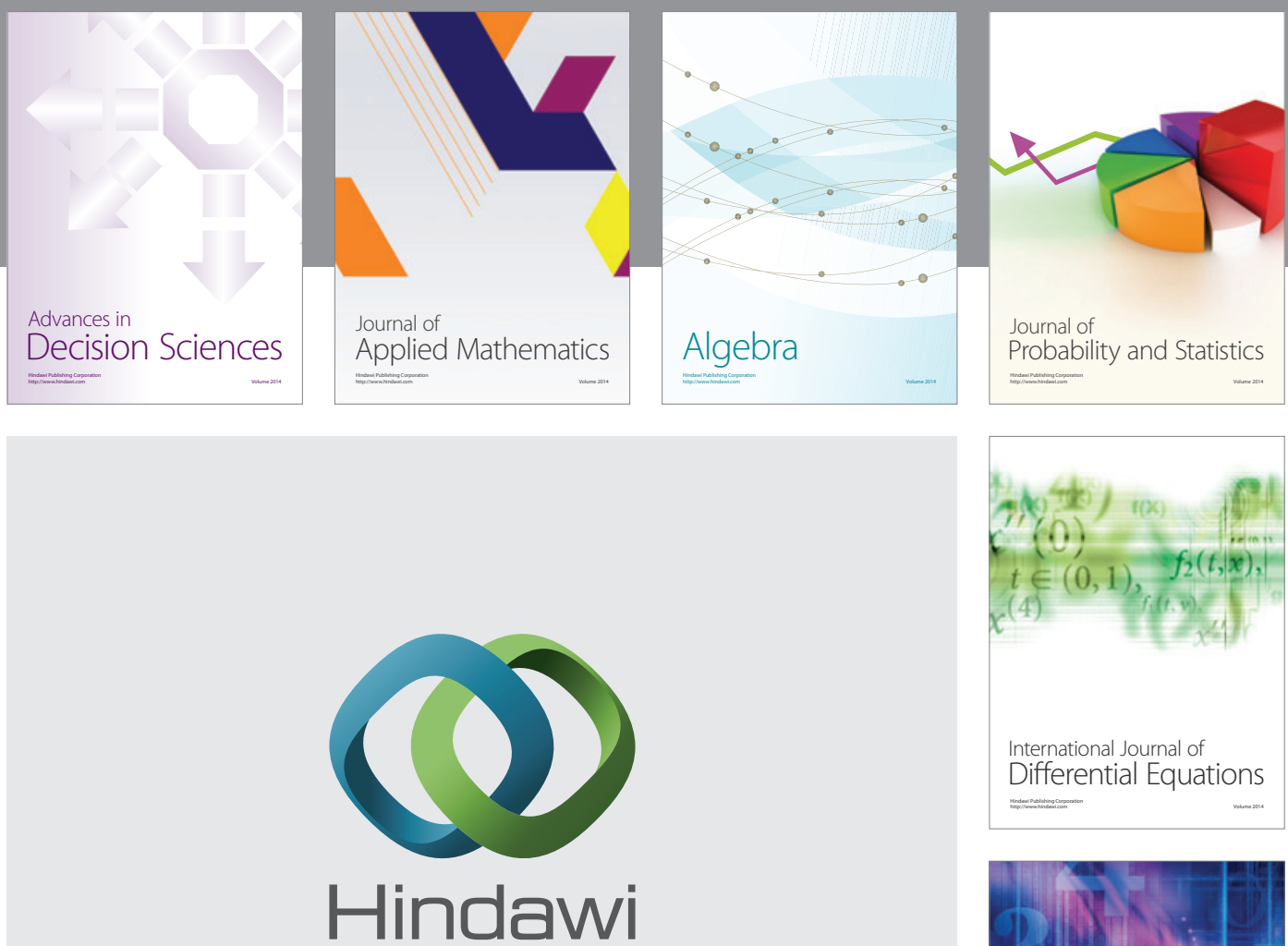

Submit your manuscripts at http://www.hindawi.com
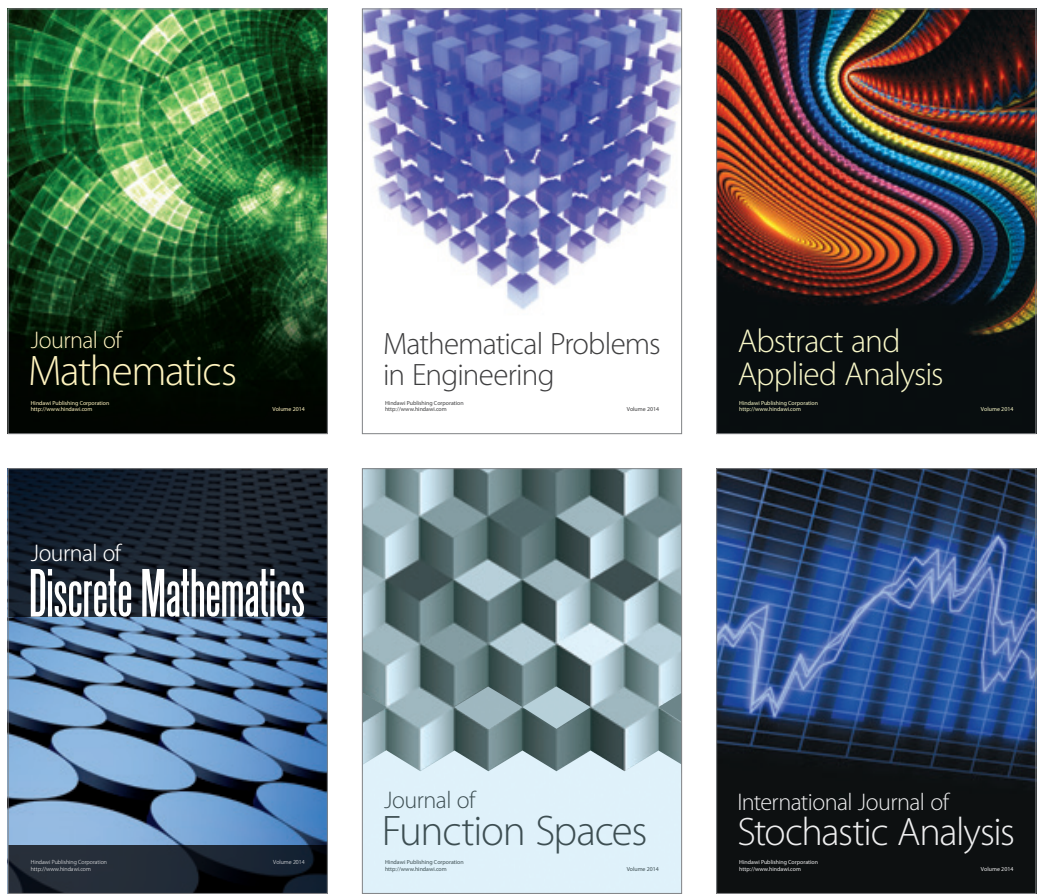

Journal of

Function Spaces

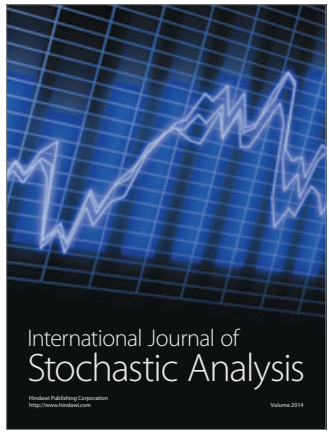

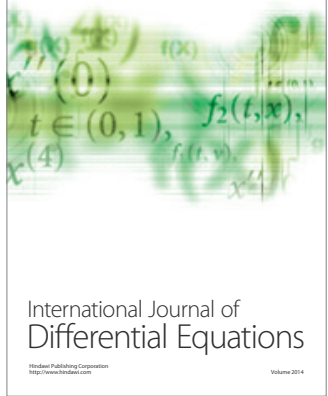
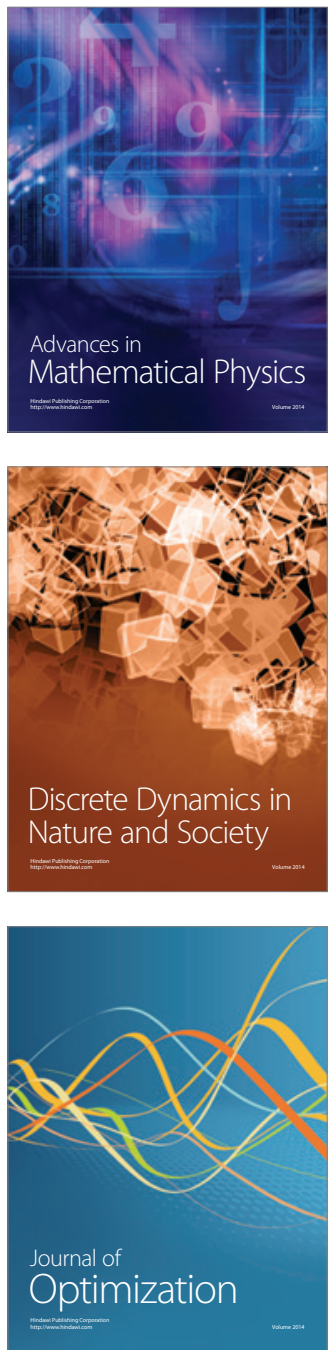